ALEA, Lat. Am. J. Probab. Math. Stat. 15, 453-478 (2018)

DOI: $10.30757 /$ ALEA.v15-19

\title{
Sample path properties of reflected Gaussian processes
}

\section{Kamil Marcin Kosiński and Peng Liu}

Mathematical Institute, University of Wrocław, pl. Grunwaldzki 2/4, 50-384 Wrocław, Poland.

E-mail address: Kamil.Kosinski@math.uni.wroc.pl

Department of Actuarial Science, University of Lausanne, UNIL-Dorigny 1015 Lausanne, Switzerland

E-mail address: Peng.Liu@unil.ch

Abstract. We consider a stationary queueing process $Q_{X}$ fed by a centered Gaussian process $X$ with stationary increments and variance function satisfying classical regularity conditions. A criterion when, for a given function $f, \mathbb{P}\left(Q_{X}(t)>f(t)\right.$ i.o. $)$ equals 0 or 1 is provided. Furthermore, an Erdös-Révész type law of the iterated logarithm is proven for the last passage time $\xi(t)=\sup \left\{s: 0 \leq s \leq t, Q_{X}(s) \geq\right.$ $f(s)\}$. Both of these findings extend previously known results that were only available for the case when $X$ is a fractional Brownian motion.

\section{Introduction and Main Results}

Let $X=\{X(t): t \geq 0\}$ be a centered Gaussian process with stationary increments and almost surely continuous sample paths. Given $c>0$, consider a reflected (at 0) Gaussian process $Q_{X}=\left\{Q_{X}(t): t \geq 0\right\}$ given by the following formula

$$
Q_{X}(t)=X(t)-c t+\max \left(Q_{X}(0),-\inf _{s \in[0, t]}(X(s)-c s)\right) .
$$

It is well known in queueing and risk theory, e.g., Reich (1958), that the unique stationary solution of (1.1) has the following representation

$$
Q_{X}(t)=\sup _{-\infty<s \leq t}(X(t)-X(s)-c(t-s)) .
$$

Due to numerous application, $Q_{X}$ has been studied in the literature under different levels of generality, e.g., Norros (1994); Hüsler and Piterbarg (1999); Dębicki (2002); Hüsler and Piterbarg (2004); Dieker (2005); Hashorva et al. (2013); Liu et al. (2015).

Received by the editors November 26th, 2017; accepted March 26th, 2018.

2010 Mathematics Subject Classification. Primary: 60F15, 60G70; Secondary: 60G22.

Key words and phrases. Extremes of Gaussian fields, storage processes, Gaussian processes, law of the iterated logarithm. 
Let $f$ be any positive nondecreasing function on $\mathbb{R}$. Kolmogorov's zero-one law implies that the process $Q_{X}$ crosses the function $f$ infinitely many times with probability 0 or 1 . Assume that $\mathbb{P}\left(Q_{X}(t)>f(t)\right.$ i.o. $)=1$ and define $\xi_{f}=\left\{\xi_{f}(t)\right.$ : $t \geq 0\}$ as the last crossing time before time $t$, that is,

$$
\xi_{f}(t)=\sup \left\{s: 0 \leq s \leq t, Q_{X}(s) \geq f(s)\right\} .
$$

By the assumption on $f$ it follows that

$$
\lim _{t \rightarrow \infty} \xi_{f}(t)=\infty \quad \text { and } \quad \limsup _{t \rightarrow \infty}\left(\xi_{f}(t)-t\right)=0 \quad \text { a.s. }
$$

The purpose of this paper is to provide a tractable criterion to verify the zeroone law as well as to give the asymptotic lower bound on $\xi_{f}(t)-t$. Erdös and Révész (1990) investigated the lower bound in the case when $Q_{X}$ is substituted by Brownian motion $W$ and $f(t)=\sqrt{2 t \log _{2} t}$ with $\log _{2} t=\log \log t$. Subsequently similar results are known as Erdös-Révész type law of the iterated logarithm.

In the reminder of the paper we impose the following assumptions on variance function $\sigma^{2}$ of $X$ :

AI: $\lim _{t \rightarrow \infty} \sigma^{2}(t) / t^{2 \alpha_{\infty}}=A_{\infty}$, for some $A_{\infty}>0, \alpha_{\infty} \in(0,1)$. Further, $\sigma^{2}$ is positive and twice continuously differentiable on $(0, \infty)$ with its first derivative $\dot{\sigma}^{2}$ and second derivative $\ddot{\sigma}^{2}$ being ultimately monotone at $\infty$.

AII: $\lim _{t \rightarrow 0^{+}} \sigma^{2}(t) / t^{2 \alpha_{0}}=A_{0}$, for some $A_{0}>0, \alpha_{0} \in(0,1]$.

Assumptions AI-AII allow us to cover models that play important role in Gaussian storage models, including both aggregations of fractional Brownian motions and integrated stationary Gaussian processes; see, e.g., Norros (1994); Hüsler and Piterbarg (1999); Dieker (2005); Dębicki (2002). In further analysis we tacitly assume that the variance function $\sigma^{2}$ of $X$ satisfies both AI and AII. Our first contribution is the following criterion; see, e.g., Watanabe (1970); Qualls and Watanabe (1971) for similar results in the classical setting of non-reflected stationary Gaussian process.

Theorem 1.1. For all positive and nondecreasing functions $f$ on some interval $[T, \infty), T>0$,

$$
\mathbb{P}\left(Q_{X}(t)>f(t) \quad \text { i.o. }\right)=0 \text { or } 1,
$$

according as the integral

$$
\int_{T}^{\infty} \frac{\psi(f(u))}{f(u)} \mathrm{d} u \quad \text { is finite or infinite, }
$$

where

$$
\psi(u):=\mathbb{P}\left(\sup _{t \in[0, u]} Q_{X}(t)>u\right) .
$$

With $\overleftarrow{m}$ being the generalized inverse of

$$
m(u)=\inf _{t \geq 0} \frac{u(1+c t)}{\sigma(u t)}
$$


define function $f_{p}$ by

$$
\begin{aligned}
& f_{p}(t)=\overleftarrow{m}\left(\sqrt{2\left(\log t+\left(\frac{\gamma-1}{2\left(1-\alpha_{\infty}\right)}-p\right) \log _{2} t\right)}\right), \\
& \gamma=\left\{\begin{array}{cl}
\frac{2\left(1-\alpha_{\infty}\right)}{\alpha_{\infty}} & \alpha_{\infty} \geq 1 / 2 \\
\frac{2\left(1+\alpha_{0}-2 \alpha_{\infty}\right)}{\alpha_{0}} & \alpha_{\infty}<1 / 2
\end{array}\right.
\end{aligned}
$$

and a positive constant $\mathscr{C}$ as

$$
\mathscr{C}=\frac{1}{2}\left(\mathcal{H}_{\eta_{\alpha_{\infty}}}\right)^{2} \sqrt{\frac{A}{B}} \zeta_{\alpha_{\infty}}\left(\frac{\sqrt{2 A_{\infty}}}{A}\right)^{\frac{\gamma-1}{1-\alpha_{\infty}}},
$$

where the remaining constants are defined in Equation 3.43. Since the exact asymptotics of $\psi(u)$, as $u$ grows large, were found in (Dębicki and Liu, 2016), c.f., Theorem 3.1, it follows that

$$
\frac{\psi\left(f_{p}(u)\right)}{f_{p}(u)}=\mathscr{C}\left(u \log ^{1-p} u\right)^{-1}(1+o(u)), \text { as } u \rightarrow \infty .
$$

Hence, by Theorem 1.1, $\mathbb{P}\left(Q_{B_{H}}(t)>f_{p}(t)\right.$ i.o. $)=1$ provided that $p \geq 0$, which leads to the following conclusion after deriving the exact asymptotics of $f_{p}$.

\section{Corollary 1.2.}

$$
\limsup _{t \rightarrow \infty} \frac{Q_{X}(t)}{(\log t)^{\frac{1}{2\left(1-\alpha_{\infty}\right)}}}=\left(\frac{2 A_{\infty}}{A^{2}}\right)^{\frac{1}{2\left(1-\alpha_{\infty}\right)}} \text { a.s. }
$$

Our second contribution is as follows.

Theorem 1.3. If $p>1$, then

$$
\liminf _{t \rightarrow \infty} \frac{\xi_{f_{p}}(t)-t}{h_{p}(t)}=-1 \text { a.s. }
$$

If $p \in(0,1]$, then

$$
\liminf _{t \rightarrow \infty} \frac{\log \left(\xi_{f_{p}}(t) / t\right)}{h_{p}(t) / t}=-1 \text { a.s. }
$$

where

$$
h_{p}(t)=p \frac{f_{p}(t)}{\psi\left(f_{p}(t)\right)} \log _{2} t
$$

Theorem 1.3 shows that for $t$ big enough, there exists an $s$ in $\left[t-h_{p}(t), t\right]$ such that $Q_{X}(s) \geq f_{p}(s)$ and that the length of the interval $h_{p}(t)$ is smallest possible. Theorem 1.1 and Theorem 1.3 generalize the main results of Dębicki and Kosiński (2017), which considered the special case when $X \equiv B_{H}$ is a fractional Brownian motion with any Hurst parameter $H \in(0,1)$; see also (Shao, 1992; Dębicki and Kosiński, 2018) for similar results for non-reflected Gaussian processes and Gaussian order statistics. The organization of the rest of paper is as follows. The notation and examples of Gaussian processes $X$ that fall under our framework are displayed in Section 2 followed by properties of the storage process $Q_{X}$ in Section 3. Section 4 gives two useful tools and some auxiliary lemmas for the proof of the main results which are presented in Section 5. 


\section{Notation and Special Cases}

We write $f(u) \sim g(u)$ if $\lim _{u \rightarrow \infty} f(u) / g(u)=1$. By $\overleftarrow{\sigma}$ we denote the generalized inverse function to $\sigma, \Psi$ denotes the tail distribution function of the standard Normal random variable. Function $f$ is ultimately monotone if there exists a constant $M>0$ such that $f$ is monotone over $(M, \infty)$. For a centered continuous Gaussian process with stationary increments $V=\{V(t): t \in \mathbb{R}\}$, such that $V(0)=0$,

$$
\operatorname{Cov}(V(t), V(s))=\frac{\sigma_{V}^{2}(t)+\sigma_{V}^{2}(s)-\sigma_{V}^{2}(t-s)}{2},
$$

we introduce the generalized Pickands' constant on a compact set $E \subset \mathbb{R}^{d}$ as

$$
\mathcal{H}_{V}(E)=\mathbb{E} \exp \left(\sup _{t \in E}\left(\sqrt{2} V(t)-\sigma_{V}^{2}(t)\right)\right) .
$$

Let

$$
\mathcal{H}_{V}=\lim _{S \rightarrow \infty} \frac{\mathcal{H}_{V}([0, S])}{S} .
$$

We refer to (Dębicki and Kosiński, 2014) for the finiteness of $\mathcal{H}_{V}(E)$ and to (Dębicki et al., 2017; Dębicki and Hashorva, 2017) for the fact that $\mathcal{H}_{V} \in(0, \infty)$. Furthermore, see (Dębicki, 2002; Dieker, 2005) for the analysis of other properties of Pickands'-type constants.

Special cases. Fractional Brownian motion. Let $B_{H}=\left\{B_{H}(t): t \geq 0\right\}$ denote fBm with Hurst index $H \in(0,1]$ which is a centered Gaussian processes with continuous sample paths and covariance function satisfying

$$
\mathbb{C o v}\left(B_{H}(t), B_{H}(s)\right)=\frac{|s|^{2 H}+|t|^{2 H}-|t-s|^{2 H}}{2}, \quad s, t \geq 0 .
$$

Direct calculations show that

$$
\begin{aligned}
& \sigma^{2}(t)=|t|^{2 H}, \quad m(u)=A u^{1-H} \\
& A=\left(\frac{H}{c(1-H)}\right)^{-H} \frac{1}{1-H}, \quad B=\left(\frac{H}{c(1-H)}\right)^{-H-2} H \\
& \overleftarrow{m}(u)=A^{-\frac{1}{1-H}} u^{\frac{1}{1-H}}, \quad f_{p}(u)=\left(\frac{2}{A^{2}}\left(\log u+\left(\frac{2-3 H}{2 H(1-H)}-p\right) \log _{2} u\right)\right)^{\frac{1}{2(1-H)}}, \\
& h_{p}(u)=p^{-1} u \log ^{1-p} u \log _{2} u, \quad \mathscr{C}=\frac{1}{2}\left(\mathcal{H}_{B_{H}}\right)^{2} \sqrt{\frac{A}{B}}\left(\frac{\sqrt{2}\left(\tau^{*}\right)^{2 H}}{1+c \tau^{*}}\right)\left(\frac{\sqrt{2}}{A}\right)^{\frac{2-3 H}{H(1-H)}}
\end{aligned}
$$

with $\tau^{*}=\frac{H}{c(1-H)}$. This coincides with (Dębicki and Kosiński, 2017, Theorem 1 and 2).

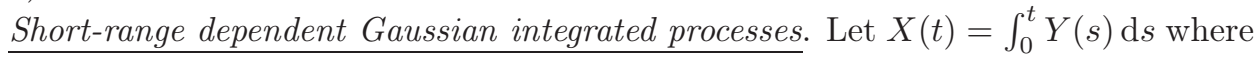
$Y$ is a centered stationary Gaussian process with unit variance and correlation function $r(t)=\mathbb{C o v}(Y(s+t), Y(s)), s \geq 0, t \geq 0$. We say that $X$ possesses shortrange dependence property if:

S1: $r$ is a continuous function on $[0, \infty)$ such that, $\lim _{t \rightarrow \infty} \operatorname{tr}(t)=0$;

S2: $r$ is decreasing over $[0, \infty)$ and $\int_{0}^{\infty} r(t) \mathrm{d} t=\frac{1}{G}$ for some $0<G<\infty$;

S3: $\int_{0}^{\infty} s^{2}|r(s)| \mathrm{d} s<\infty$. 
The above assumptions go line by line the same as the assumptions in Debicki (2002) except a little modification. S1-S3 cover wide range of stationary Gaussian processes such as the process with correlation function

$$
r(t)=e^{-|t|^{\alpha}}, \quad \alpha \in(0,2] .
$$

In particular if $r(t)=e^{-|t|}, X$ is the so-called Ornstein-Uhlenbeck process. Apparently, if $\mathbf{S 1 - S 3}$ are satisfied, then

$$
\sigma^{2}(t)=2 \int_{0}^{t} \int_{0}^{s} r(v) \mathrm{d} v \mathrm{~d} s
$$

satisfies AI-AII. Note that

$$
\sigma^{2}(t) \sim t^{2}, \quad \text { as } t \rightarrow 0, \quad \sigma^{2}(t) \sim \frac{2}{G} t, \text { as } t \rightarrow \infty .
$$

Dębicki (2002, Proposition 6.1) shows that

$$
m^{2}(u)=2 G u+2 G^{2} G_{1}+o(1), \text { as } u \rightarrow \infty,
$$

with $G_{1}=\int_{0}^{\infty} \operatorname{tr}(t) \mathrm{d} t$. This indicates that $m(u)$ can be replaced by $\widehat{m}(u)=$ $\sqrt{2 G u+2 G^{2} G_{1}}$ in Theorem 3.1 and Theorem 1.3. Under this replacement, we have that

$$
\overleftarrow{\widehat{m}(u)}=\frac{u^{2}}{2 G}-G G_{1}, \quad f_{p}(t)=\frac{1}{G}\left(\log t+(1-p) \log _{2} t\right)-G G_{1}
$$

and

$$
h_{p}(u)=p \mathscr{C}^{-1} u \log ^{1-p} u \log _{2} u, \quad \mathscr{C}=\frac{2\left(\mathcal{H}_{\eta_{1 / 2}}\right)^{2}\left(\overleftarrow{\sigma}\left(\frac{\sqrt{2}}{c G}\right)\right)^{-2}}{A^{3 / 2} \sqrt{B} G}
$$

with $\eta_{1 / 2}=\frac{c G}{\sqrt{2}} X\left(\overleftarrow{\sigma}\left(\frac{\sqrt{2}}{c G}\right) t\right), A=2 c^{1 / 2}$, and $B=\frac{1}{2} c^{5 / 2}$

\section{Properties of the storage process}

Before we present our auxiliary results, we need to introduce some notation and state some properties of the supremum of the process $Q_{X}$ as derived in (Piterbarg, 2001; Hüsler and Piterbarg, 2004). We begin with the relation

$$
\mathbb{P}\left(\sup _{t \in[0, T]} Q_{X}(t)>u\right)=\mathbb{P}\left(\sup _{\substack{s \in[0, T / u] \\ \tau \geq 0}} Z_{u}(s, \tau)>m(u)\right), \quad \text { for any } T>0,
$$

where

$$
Z_{u}(s, \tau)=\frac{X(u(\tau+s))-X(u s)}{u(1+c \tau)} m(u) .
$$

Note that $Z_{u}(s, \tau)$ is a Gaussian field, stationary in $s$, but not in $\tau$. The variance $\sigma_{u}^{2}(\tau)$ of $Z_{u}(s, \tau)$ equals $\frac{\sigma^{2}(u \tau)}{(u(1+c \tau))^{2}} m^{2}(u)$ and $\sigma_{u}(\tau)$ has a single maximum point at $\tau(u)$ for $u$ sufficiently large with $\lim _{u \rightarrow \infty} \tau(u)=\tau^{*}$, where

$$
\tau^{*}=\frac{\alpha_{\infty}}{c\left(1-\alpha_{\infty}\right)} .
$$

Taylor's formula shows that, for each $u>0$ sufficiently large,

$$
\sigma_{u}(\tau)=\sigma_{u}(\tau(u))+\dot{\sigma}_{u}(\tau(u))(\tau-\tau(u))+\frac{1}{2} \ddot{\sigma}_{u}(\xi)(\tau-\tau(u))^{2}
$$


with $\xi \in(\tau, \tau(u))$. Noting that $\sigma_{u}(\tau(u))=1$, for $u$ sufficiently large, $\dot{\sigma}_{u}(\tau(u))=0$ and for $\lim _{u \rightarrow \infty} \delta_{u}=0$

$$
\lim _{u \rightarrow \infty} \sup _{|\tau-\tau(u)|<\delta_{u}}\left|\frac{1}{2} \ddot{\sigma}_{u}(\xi)-\frac{B}{2 A}\right|=0
$$

we have

$$
\lim _{u \rightarrow \infty} \sup _{\tau \neq \tau(u),|\tau-\tau(u)|<\delta_{u}}\left|\frac{1-\sigma_{u}(\tau)}{\frac{B}{2 A}(\tau-\tau(u))^{2}}-1\right|=0
$$

with $\lim _{u \rightarrow \infty} \delta_{u}=0$, where

$$
A=\left(\frac{\alpha_{\infty}}{c\left(1-\alpha_{\infty}\right)}\right)^{-\alpha_{\infty}} \frac{1}{1-\alpha_{\infty}}, \quad B=\left(\frac{\alpha_{\infty}}{c\left(1-\alpha_{\infty}\right)}\right)^{-\alpha_{\infty}-2} \alpha_{\infty} .
$$

Let $r_{u, u^{\prime}}\left(s, \tau, s^{\prime}, \tau^{\prime}\right)$ be the correlation function of $Z_{u}(s, \tau)$ and $Z_{u^{\prime}}\left(s^{\prime}, \tau^{\prime}\right)$. Then

$$
\begin{aligned}
r_{u, u^{\prime}}\left(s, \tau, s^{\prime}, \tau^{\prime}\right) & =\left(2 \sigma(u \tau) \sigma\left(u^{\prime} \tau^{\prime}\right)\right)^{-1} \cdot\left(-\sigma^{2}\left(\left|u s-u^{\prime} s^{\prime}+u \tau-u^{\prime} \tau^{\prime}\right|\right)\right. \\
& \left.+\sigma^{2}\left(\left|u s-u^{\prime} s^{\prime}+u \tau\right|\right)+\sigma^{2}\left(\left|u s-u^{\prime} s^{\prime}-u^{\prime} \tau^{\prime}\right|\right)-\sigma^{2}\left(\left|u s-u^{\prime} s^{\prime}\right|\right)\right) .
\end{aligned}
$$

Denote by

$$
r_{u}\left(s, \tau, s^{\prime}, \tau^{\prime}\right)=r_{u, u}\left(s, \tau, s^{\prime}, \tau^{\prime}\right) .
$$

Then Lemma 5.4 in Dębicki and Liu (2016) gives that, with $\delta_{u}>0$ and $\lim _{u \rightarrow \infty} \delta_{u}=$ 0 ,

$$
\lim _{u \rightarrow \infty} \sup _{(s, \tau) \neq\left(s^{\prime}, \tau^{\prime}\right),|\tau-\tau(u)|,\left|\tau^{\prime}-\tau(u)\right|,\left|s-s^{\prime}\right| \leq \delta_{u}}\left|\frac{1-r_{u}\left(s, \tau, s^{\prime}, \tau^{\prime}\right)}{\frac{\sigma^{2}\left(u\left|s-s^{\prime}+\tau-\tau^{\prime}\right|\right)+\sigma^{2}\left(u\left|s-s^{\prime}\right|\right)}{2 \sigma^{2}\left(u \tau^{*}\right)}}-1\right|=0 .
$$

Now assume that

$$
\frac{u \tau+u^{\prime} \tau^{\prime}}{\left|u s-u^{\prime} s^{\prime}\right|}<\frac{1}{2}
$$

and without loss of generality, us $>u^{\prime} s^{\prime}$. Then Taylor's formula gives that

$$
r_{u, u^{\prime}}\left(s, \tau, s^{\prime}, \tau^{\prime}\right)=\frac{-\ddot{\sigma^{2}}\left(\left|u s-u^{\prime} s^{\prime}+v_{1}-v_{2}\right|\right) u \tau u^{\prime} \tau^{\prime}}{2 \sigma(u \tau) \sigma\left(u^{\prime} \tau^{\prime}\right)},
$$

with $v_{1} \in(0, u \tau), v_{2} \in\left(0, u^{\prime} \tau^{\prime}\right)$. Noting that by (3.6)

$$
\left|u s-u^{\prime} s^{\prime}+v_{1}-v_{2}\right| \geq u \tau+u^{\prime} \tau,
$$

in light of (Bingham et al., 1987, Theorem 1.7.2) and by AI-AII we have $\frac{\left|u s-u^{\prime} s^{\prime}+v_{1}-v_{2}\right|^{2} \ddot{\sigma}^{2}\left(\left|u s-u^{\prime} s^{\prime}+v_{1}-v_{2}\right|\right)}{\sigma^{2}\left(\left|u s-u^{\prime} s^{\prime}+v_{1}-v_{2}\right|\right)} \rightarrow 2 \alpha_{\infty}\left(2 \alpha_{\infty}-1\right)$, as $u \tau, u^{\prime} \tau^{\prime} \rightarrow \infty$.

Hence

$$
r_{u, u^{\prime}}\left(s, \tau, s^{\prime}, \tau^{\prime}\right) \sim-\alpha_{\infty}\left(2 \alpha_{\infty}-1\right)\left|\frac{\sqrt{u \tau u^{\prime} \tau^{\prime}}}{u s-u^{\prime} s^{\prime}+v_{1}-v_{2}}\right|^{2 \lambda}, \text { as } u \tau, u^{\prime} \tau^{\prime} \rightarrow \infty,
$$


where $\lambda=1-\alpha_{\infty}>0$. This implies that for any $0<\epsilon<\frac{1}{2}$ if

$$
\frac{u \tau+u^{\prime} \tau^{\prime}}{\left|u s-u^{\prime} s^{\prime}\right|}<\epsilon
$$

then, for $u \tau$ and $u \tau^{\prime}$ both sufficiently large,

$$
\left|r_{u, u^{\prime}}\left(s, \tau, s^{\prime}, \tau^{\prime}\right)\right| \leq(1-2 \epsilon)^{2\left(\alpha_{\infty}-1\right)}\left|\frac{\sqrt{u \tau u^{\prime} \tau^{\prime}}}{u s-u^{\prime} s^{\prime}}\right|^{2 \lambda} .
$$

Next we focus on the case when $u \sim u^{\prime},\left|s-s^{\prime}\right| \leq M$ and $\left|\tau-\tau_{0}\right|,\left|\tau^{\prime}-\tau^{*}\right| \leq \delta\left(u, u^{\prime}\right)$ with $\tau^{*}$ defined in (3.2) and $\lim _{u, u^{\prime} \rightarrow \infty} \delta\left(u, u^{\prime}\right)=0$. In light of AI and AII, noting that $\sigma^{2}$ is bounded over any compact interval, using uniform convergence theorem in Bingham et al. (1987) we have that, for $u \sim u^{\prime}$,

$$
\begin{aligned}
& \lim _{u, u^{\prime} \rightarrow \infty} \sup _{\left|s-s^{\prime}\right| \leq M,\left|\tau-\tau^{*}\right|,\left|\tau^{\prime}-\tau^{*}\right| \leq \delta\left(u, u^{\prime}\right)} \mid \frac{\sigma^{2}\left(\left|u s-u^{\prime} s^{\prime}+u \tau\right|\right)+\sigma^{2}\left(\left|u s-u^{\prime} s^{\prime}-u^{\prime} \tau^{\prime}\right|\right)}{\sigma^{2}(u)} \\
& -\left|s-s^{\prime}+\tau^{*}\right|^{2 \alpha_{\infty}}-\left|s-s^{\prime}-\tau^{*}\right|^{2 \alpha_{\infty}} \mid=0, \\
& \lim _{u, u^{\prime} \rightarrow \infty} \sup _{\left|s-s^{\prime}\right| \leq M,\left|\tau-\tau^{*}\right|,\left|\tau^{\prime}-\tau^{*}\right| \leq \delta\left(u, u^{\prime}\right)} \mid \frac{\sigma^{2}\left(\left|u s-u^{\prime} s^{\prime}+u \tau-u^{\prime} \tau^{\prime}\right|\right)+\sigma^{2}\left(\left|u s-u^{\prime} s^{\prime}\right|\right)}{\sigma^{2}(u)} \\
& -2\left|s-s^{\prime}\right|^{2 \alpha_{\infty}} \mid=0, \\
& \left.\lim _{u, u^{\prime} \rightarrow \infty} \sup _{\left|s-s^{\prime}\right| \leq M,\left|\tau-\tau^{*}\right|,\left|\tau^{\prime}-\tau^{*}\right| \leq \delta\left(u, u^{\prime}\right)}\left|\frac{\sigma(u \tau) \sigma\left(u \tau^{\prime}\right)}{\sigma^{2}(u)}-\right| \tau^{*}\right|^{2 \alpha_{\infty}} \mid=0 .
\end{aligned}
$$

Hence for $u \sim u^{\prime}$

$$
\lim _{u, u^{\prime} \rightarrow \infty} \sup _{\left|s-s^{\prime}\right| \leq M,\left|\tau-\tau^{*}\right|,\left|\tau^{\prime}-\tau^{*}\right| \leq \delta\left(u, u^{\prime}\right)}\left|r_{u, u^{\prime}}\left(s, \tau, s^{\prime}, \tau^{\prime}\right)-g\left(s-s^{\prime}\right)\right|=0,
$$

with

$$
g(t)=\frac{\left|t+\tau^{*}\right|^{2 \alpha_{\infty}}+\left|t-\tau^{*}\right|^{2 \alpha_{\infty}}-2|t|^{2 \alpha_{\infty}}}{2\left(\tau^{*}\right)^{2 \alpha_{\infty}}} .
$$

Note that $g(0)=1$ and for any $0<\delta<1$, there exists $0<c_{\delta}<1 / 2$ such that

$$
\inf _{|t|<c_{\delta}} g(t)>\delta, \quad \sup _{|t|>\delta} g(t)<1-c_{\delta} .
$$

The proof of (3.10) is postponed to Appendix. Following (3.9) and (3.10), we have that with $u \sim u^{\prime}$, for $u$ sufficiently large,

$$
\begin{aligned}
& \inf _{\left|s-s^{\prime}\right|<c \delta,\left|\tau-\tau^{*}\right|,\left|\tau^{\prime}-\tau^{*}\right| \leq \delta\left(u, u^{\prime}\right)} r_{u, u^{\prime}}\left(s, \tau, s^{\prime}, \tau^{\prime}\right)>\delta / 2, \\
& \sup _{\left|s-s^{\prime}\right|>\delta,\left|\tau-\tau^{*}\right|,\left|\tau^{\prime}-\tau^{*}\right| \leq \delta\left(u, u^{\prime}\right)} r_{u, u^{\prime}}\left(s, \tau, s^{\prime}, \tau^{\prime}\right)<1-c_{\delta} / 2<1 .
\end{aligned}
$$

3.1. Asymptotics. Let $\tau^{*}(u)=(\log m(u)) / m(u)$ and $J(u)=\{\tau:|\tau-\tau(u)| \leq$ $\left.\tau^{*}(u)\right\}$. Due to the following lemma, while analyzing tail asymptotics of the supremum of $Z_{u}$, we can restrict the considered domain of $(s, \tau)$ to a strip $J(u)$.

Lemma 3.1 (Dębicki and Liu (2016), Lemma 5.6 and Theorem 3.3). There exists a positive constant $C$ such that for any $v, T>0$,

$$
\mathbb{P}\left(\sup _{(s, \tau) \in[0, T] \times(J(u))^{c}} Z_{u}(s, \tau)>m(u)\right) \leq C T \frac{u^{\gamma}}{m(u)} \Psi(m(u)) \exp \left(-\frac{b}{4} \log ^{2}(m(u))\right),
$$


where $b=B /(2 A)$. Furthermore, for any $T>0$ such that, there exist $c \in\left(0, \frac{1}{2}\right)$ and $H^{\prime} \in(-\gamma / 2,0)$, such that $u^{H^{\prime}}<T<\exp \left(\mathrm{cm}^{2}(u)\right)$ for $u$ sufficiently large,

$\mathbb{P}\left(\sup _{(s, \tau) \in[0, T] \times J(u)} Z_{u}(s, \tau)>m(u)\right)=\left(\mathcal{H}_{\eta_{\alpha_{\infty}}}\right)^{2} \sqrt{\frac{2 A \pi}{B}} \zeta_{\alpha_{\infty}} T \frac{u^{\gamma}}{m(u)} \Psi(m(u))(1+o(1))$,

where

$$
\begin{aligned}
\eta_{\alpha_{\infty}}(t)=\left\{\begin{array}{cc}
B_{\alpha_{\infty}}(t) & \alpha_{\infty}>1 / 2 \\
\frac{1+c \tau^{*}}{\sqrt{2} A_{\infty} \tau^{*}} X\left(\overleftarrow{\sigma}\left(\frac{\sqrt{2} A_{\infty} \tau^{*}}{1+c \tau^{*}}\right) t\right) & \alpha_{\infty}=1 / 2 \\
B_{\alpha_{0}}(t) & \alpha_{\infty}<1 / 2
\end{array}\right. \\
\zeta_{\alpha_{\infty}}=\left\{\begin{array}{cc}
\left(\frac{\sqrt{2 A_{\infty}}\left(\tau^{*}\right)^{2 \alpha_{\infty}}}{1+c \tau^{*}}\right)^{-2 / \alpha_{\infty}} & \alpha_{\infty}>1 / 2 \\
\left(\overleftarrow{\sigma}\left(\frac{\sqrt{2} A_{\infty} \tau^{*}}{1+c \tau^{*}}\right)\right)^{-2} & \alpha_{\infty}=1 / 2 \\
\left(\frac{\sqrt{2} A_{\infty}\left(\tau^{*}\right)_{\infty}^{2 \alpha_{\infty}}}{\sqrt{A_{0}}\left(1+c \tau^{*}\right)}\right)^{-2 / \alpha_{0}} & \alpha_{\infty}<1 / 2
\end{array}\right.
\end{aligned}
$$

with $\gamma$ defined in (1.2) and $\tau^{*}$ given by (3.2).

3.2. Discretization. For a fixed $T, \theta>0$ and some $u>0$, let us define a discretization of the set $[0, T] \times J(u)$ as follows

$$
\begin{gathered}
s_{l}=l q(u), 0 \leq l \leq L, L=[T / q(u)], q(u)=\theta \frac{\Delta(u)}{u}, \Delta(u)=\overleftarrow{\sigma}\left(\frac{\sqrt{2} \sigma^{2}\left(u \tau^{*}\right)}{u\left(1+c \tau^{*}\right)}\right) \\
\tau_{n}=\tau(u)+n q(u), 0 \leq|n| \leq N, N=\left[\tau^{*}(u) / q(u)\right], E_{l, n}(u)=\left[s_{l}, s_{l+1}\right] \times\left[\tau_{n}, \tau_{n+1}\right] .
\end{gathered}
$$

Along the similar lines as in (Hüsler and Piterbarg, 2004, Lemma 6) we get the following lemma.

Lemma 3.2. There exist positive constants $K_{1}, K_{2}, u_{0}>0$, such that, for any $\theta=\theta(u)>0$ with $\lim _{u \rightarrow \infty} \theta(u)=0, u \geq u_{0}$ and $\eta \in\left(0, \min \left(\alpha_{0}, \alpha_{\infty}\right)\right)$

$$
\begin{aligned}
& \mathbb{P}\left(\max _{\substack{0 \leq l \leq L \\
0 \leq|n| \leq N}} Z_{u}\left(s_{l}, \tau_{n}\right) \leq m(u)-\frac{\theta^{\eta}}{m(u)}, \sup _{\substack{s \in[0, T] \\
\tau \in J(u)}} Z_{u}(s, \tau)>m(u)\right) \\
& \leq K_{1} \frac{u^{\gamma}}{m(u)} \Psi(m(u)) e^{-\frac{\theta^{-2 H}}{K_{2}}}
\end{aligned}
$$

with $H \in\left(0, \min \left(\alpha_{0}, \alpha_{\infty}\right)-\eta\right)$.

Proof: Conditioning on $Z_{u}\left(s_{l}, \tau_{n}\right)=m(u)-\frac{\theta^{\eta}}{m(u)}$, we have for $u$ sufficiently large

$$
\begin{aligned}
& \mathbb{P}\left(Z_{u}\left(s_{l}, \tau_{n}\right) \leq\right.\left.m(u)-\frac{\theta^{\eta}}{m(u)}, \sup _{(s, \tau) \in E_{l, n}(u)} Z_{u}(s, \tau)>m(u)\right) \\
&=\int_{\theta^{\eta}}^{\infty} \frac{1}{\sqrt{2 \pi} m(u) \sigma_{u}\left(\tau_{n}\right)} e^{-\frac{(m(u)-y / m(u))^{2}}{2 \sigma_{u}^{2}\left(\tau_{n}\right)}} \\
& \quad \times \mathbb{P}\left(\sup _{(s, \tau) \in E_{l, n}(u)} Z_{u}(s, \tau)>m(u) \mid Z_{u}\left(s_{l}, \tau_{n}\right)=m(u)-\frac{y}{m(u)}\right) \mathrm{d} y
\end{aligned}
$$




$$
\begin{aligned}
& \leq \frac{K}{\sqrt{2 \pi} m(u)} e^{-\frac{m^{2}(u)}{2 \sigma_{u}^{2}\left(\tau_{n}\right)}} \int_{\theta^{\eta}}^{\infty} e^{2 y} \\
& \quad \times \mathbb{P}\left(\sup _{(s, \tau) \in E_{l, n}(u)} Z_{u}(s, \tau)-m(u)>0 \mid Z_{u}\left(s_{l}, \tau_{n}\right)=m(u)-\frac{y}{m(u)}\right) \mathrm{d} y .
\end{aligned}
$$

Moreover,

$$
Z_{u}(s, \tau)-m(u) \mid Z_{u}\left(s_{l}, \tau_{n}\right)=m(u)-\frac{y}{m(u)} \stackrel{d}{=} Y_{u}(s, \tau)+h(u, y),
$$

holds for $(s, \tau) \in E_{l, n}(u)$, where

$$
\begin{aligned}
Y_{u}(s, \tau) & =Z_{u}(s, \tau)-r_{u}\left(s, \tau, s_{l}, \tau_{n}\right) \frac{\sigma_{u}(\tau)}{\sigma_{u}\left(\tau_{n}\right)} Z_{u}\left(s_{l}, \tau_{n}\right), \\
h(u, y) & =r_{u}\left(s, \tau, s_{l}, \tau_{n}\right) \frac{\sigma_{u}(\tau)}{\sigma_{u}\left(\tau_{n}\right)}\left(m(u)-\frac{y}{m(u)}\right)-m(u) .
\end{aligned}
$$

Taylor's formula gives that

$$
\begin{aligned}
m(u) h(u, y) & =-m^{2}(u)\left(1-r_{u}\left(s, \tau, s_{l}, \tau_{n}\right)\right)-m^{2}(u) r_{u}\left(s, \tau, s_{l}, \tau_{n}\right)\left(1-\frac{\sigma_{u}(\tau)}{\sigma_{u}\left(\tau_{n}\right)}\right) \\
& -r_{u}\left(s, \tau, s_{l}, \tau_{n}\right) \frac{\sigma_{u}(\tau)}{\sigma_{u}\left(\tau_{n}\right)} y \\
& \leq-m^{2}(u) r_{u}\left(s, \tau, s_{l}, \tau_{n}\right) \frac{\dot{\sigma}_{u}(\tau)\left(\tau_{n}-\tau\right)+(1 / 2) \ddot{\sigma}_{u}(v)\left(\tau-\tau_{n}\right)^{2}}{\sigma_{u}\left(\tau_{n}\right)} \\
& -r_{u}\left(s, \tau, s_{l}, \tau_{n}\right) \frac{\sigma_{u}(\tau)}{\sigma_{u}\left(\tau_{n}\right)} y,
\end{aligned}
$$

with $v \in\left(\tau_{n}, \tau\right)$. Using the fact that $\dot{\sigma}_{u}(\tau(u))=0$ and $\sup _{\tau \in J(u)}\left|\ddot{\sigma}_{u}(\tau)\right| \leq \frac{2 B}{A}$ for $u$ sufficiently large, by Taylor's formula, we have

$$
\begin{aligned}
m^{2}(u)\left|\dot{\sigma}_{u}(\tau)\left(\tau_{n}-\tau\right)\right| & =m^{2}(u)\left|\left(\dot{\sigma}_{u}(\tau)-\dot{\sigma}_{u}(\tau(u))\right)\left(\tau_{n}-\tau\right)\right| \\
& =m^{2}(u)\left|\tau_{n}-\tau \| \ddot{\sigma}\left(v_{1}\right)(\tau(u)-\tau)\right| \\
& \leq \frac{2 B}{A} m^{2}(u) q(u) \tau^{*}(u) \\
& =\theta \frac{2 B}{A} \frac{m(u) \Delta(u)}{u} \log m(u),
\end{aligned}
$$

with $v_{1} \in(\tau, \tau(u))$. Note that by AI-AII $\frac{m(u) \Delta(u)}{u} \log m(u) \sim \mathbb{Q} u^{v_{2}} \log u, \quad$ with $\quad v_{2}=\left\{\begin{array}{cc}2-\alpha_{\infty}-1 / \alpha_{\infty} & \alpha_{\infty} \geq 1 / 2 \\ \frac{2 \alpha_{\infty}-1}{\alpha_{0}}-\alpha_{\infty} & \alpha_{\infty}<1 / 2\end{array}\right.$.

Since $v_{2}<0$ for all $\alpha_{\infty} \in(0,1]$, then

$$
\begin{gathered}
m^{2}(u)\left|\dot{\sigma}_{u}(\tau)\left(\tau_{n}-\tau\right)\right|=o(\theta), \quad u \rightarrow \infty . \\
m^{2}(u)\left|\ddot{\sigma}_{u}(\theta)\left(\tau-\tau_{n}\right)^{2}\right| \leq K\left(\frac{m(u) \Delta(u)}{u}\right)^{2} \theta^{2}=o\left(\theta^{2}\right) .
\end{gathered}
$$

Due to the fact that $y \geq \theta^{\eta}$ with $0<\eta<1$, we have

$$
h(u, y) \leq-y(1+o(1)) .
$$


Consequently, for $u$ sufficiently large

$$
\begin{aligned}
& \mathbb{P}\left(\sup _{(s, \tau) \in E_{l, n}(u)} Z_{u}(s, \tau)-m(u)>0 \mid Z_{u}\left(s_{l}, \tau_{n}\right)=m(u)-\frac{y}{m(u)}\right) \\
& \quad \leq \mathbb{P}\left(\sup _{(s, \tau) \in[0,1]^{2}} m(u) \frac{Y_{u}\left(s_{l}+q s, \tau_{n}+q \tau\right)}{\sigma_{u}\left(\tau_{n}+q \tau\right)}>\frac{y}{\sup _{\tau \in[0,1]} \sigma_{u}\left(\tau_{n}+q \tau\right)}(1+o(1))\right) \\
& \quad \leq \mathbb{P}\left(\sup _{(s, \tau) \in[0,1]^{2}} m(u) \frac{Y_{u}\left(s_{l}+q s, \tau_{n}+q \tau\right)}{\sigma_{u}\left(\tau_{n}+q \tau\right)}>\frac{y}{2}\right)
\end{aligned}
$$

By (3.5) for $u$ large enough

$$
\begin{aligned}
& m^{2}(u) \operatorname{Var}\left(\frac{Y_{u}\left(s_{l}+q s, \tau_{n}+q \tau\right)}{\sigma_{u}\left(\tau_{n}+q \tau\right)}-\frac{Y_{u}\left(s_{l}+q s^{\prime}, \tau_{n}+q \tau^{\prime}\right)}{\sigma_{u}\left(\tau_{n}+q \tau^{\prime}\right)}\right) \\
& \leq 8 m^{2}(u)\left(1-r_{u}\left(s_{l}+q s, \tau_{n}+q \tau, s_{l}+q s^{\prime}, \tau_{n}+q \tau^{\prime}\right)\right) \\
& \leq 16 m^{2}(u) \frac{\sigma^{2}\left(u q(u)\left|s-s^{\prime}\right|\right)+\sigma^{2}\left(u q(u)\left|s+\tau-s^{\prime}-\tau^{\prime}\right|\right)}{2 \sigma^{2}\left(u \tau^{*}\right)} \\
& \leq K \frac{\sigma^{2}\left(\Delta(u) \theta\left|s-s^{\prime}\right|\right)+\sigma^{2}\left(\Delta(u) \theta\left|s+\tau-s^{\prime}-\tau^{\prime}\right|\right)}{\sigma^{2}(\Delta(u))} \\
& \leq K\left(\frac{h\left(\Delta(u) \theta\left|s-s^{\prime}\right|\right)}{h(\Delta(u))} \theta^{2 \eta^{\prime}}\left|s-s^{\prime}\right|^{2 \eta^{\prime}}\right. \\
& \left.\quad+\frac{h\left(\Delta(u) \theta\left|s+\tau-s^{\prime}-\tau^{\prime}\right|\right)}{h(\Delta(u))} \theta^{2 \eta^{\prime}}\left|s+\tau-s^{\prime}-\tau^{\prime}\right|^{2 \eta^{\prime}}\right) \\
& \leq K\left(\frac{h\left(\Delta(u) \theta\left|s-s^{\prime}\right|\right)}{h(\Delta(u))}+\frac{h\left(\Delta(u) \theta\left|s+\tau-s^{\prime}-\tau^{\prime}\right|\right)}{h(\Delta(u))}\right) \theta^{2 \eta^{\prime}}\left(\left|s-s^{\prime}\right|^{2 \eta^{\prime}}+\left|\tau-\tau^{\prime}\right|^{2 \eta^{\prime}}\right),
\end{aligned}
$$

with $s, s^{\prime}, \tau, \tau^{\prime} \in[0,1]$,

where $h(t)=\frac{\sigma^{2}(t)}{t^{2 \eta^{\prime}}}$ and $\eta^{\prime} \in\left(\eta, \min \left(\alpha_{0}, \alpha_{\infty}\right)\right)$. Then it follows from AI and AII that $h(t)>0, t>0$ is a regularly varying function at both 0 and $\infty$ with indices $2\left(\alpha_{0}-\eta^{\prime}\right)>0$ and $2\left(\alpha_{\infty}-\eta^{\prime}\right)>0$ respectively; see Bingham et al. (1987) for the definition and properties of regularly varying functions. Next we focus on the boundedness of $\sup _{s, s^{\prime} \in[0,1]} \frac{h\left(\Delta(u) \theta\left|s-s^{\prime}\right|\right)}{h(\Delta(u))}$. If $\lim _{u \rightarrow \infty} \Delta(u)=\infty$, noting that $h$ is bounded over any compact interval, then uniform convergence theorem in Bingham et al. (1987) gives that

$$
\lim _{u \rightarrow \infty} \sup _{s, s^{\prime} \in[0,1]}\left|\frac{h\left(\Delta(u) \theta\left|s-s^{\prime}\right|\right)}{h(\Delta(u))}-\left(\theta\left|s-s^{\prime}\right|\right)^{2\left(\alpha_{\infty}-\eta^{\prime}\right)}\right|=0,
$$

implying that there exists $K_{1}>0$ such that for $u$ large enough

$$
\sup _{s, s^{\prime} \in[0,1]} \frac{h\left(\Delta(u) \theta\left|s-s^{\prime}\right|\right)}{h(\Delta(u))}<K_{1} .
$$

For the case $\lim _{u \rightarrow \infty} \Delta(u)=0$, uniform convergence theorem in Bingham et al. (1987) can similarly show that the above argument holds. For $\lim _{u \rightarrow \infty} \Delta(u) \in$ $(0, \infty)$, it is obvious that

$$
\lim _{u \rightarrow \infty} \sup _{s, s^{\prime} \in[0,1]} h\left(\Delta(u) \theta\left|s-s^{\prime}\right|\right)=0, \quad \lim _{u \rightarrow \infty} h(\Delta(u)) \in(0, \infty) .
$$


Thus the boundedness of $\sup _{s, s^{\prime} \in[0,1]} \frac{h\left(\Delta(u) \theta\left|s-s^{\prime}\right|\right)}{h(\Delta(u))}$ also holds. The boundedness of $\sup _{s, s^{\prime}, \tau, \tau^{\prime} \in[0,1]} \frac{h\left(\Delta(u) \theta\left|s+\tau-s^{\prime}-\tau^{\prime}\right|\right)}{h(\Delta(u))}$ can be given similarly. Thus we have that

$$
\begin{aligned}
& m^{2}(u) \operatorname{Var}\left(\frac{Y_{u}\left(s_{l}+q s, \tau_{n}+q \tau\right)}{\sigma_{u}\left(\tau_{n}+q \tau\right)}-\frac{Y_{u}\left(s_{l}+q s^{\prime}, \tau_{n}+q \tau^{\prime}\right)}{\sigma_{u}\left(\tau_{n}+q \tau^{\prime}\right)}\right) \\
& \leq K \theta^{2 \eta^{\prime}}\left(\left|s-s^{\prime}\right|^{2 \eta^{\prime}}+\left|\tau-\tau^{\prime}\right|^{2 \eta^{\prime}}\right), \quad s, s^{\prime}, \tau, \tau^{\prime} \in[0,1],
\end{aligned}
$$

with $\eta^{\prime} \in\left(\eta, \min \left(\alpha_{0}, \alpha_{\infty}\right)\right)$. Similarly

$$
\sup _{s, \tau \in[0,1]} m^{2}(u) \operatorname{Var}\left(\frac{Y_{u}\left(s_{l}+q s, \tau_{n}+q \tau\right)}{\sigma_{u}\left(\tau_{n}+q \tau\right)}\right) \leq K \theta^{2 \eta^{\prime}} .
$$

Hence in light of Piterbarg inequality (Piterbarg, 1996, Theorem 8.1 or Dębicki and Liu, 2016, Lemma 5.1), we have for $u$ sufficiently large

$$
\begin{aligned}
& \mathbb{P}\left(\sup _{(s, \tau) \in[0,1]^{2}} m(u) \frac{Y_{u}\left(s_{l}+q s, \tau_{n}+q \tau\right)}{\sigma_{u}\left(\tau_{n}+q \tau\right)}>\frac{y}{2}\right) \\
& \mathbb{P}\left(\sup _{(s, \tau) \in[0,1]^{2}} \theta^{-\eta^{\prime}} m(u) \frac{Y_{u}\left(s_{l}+q s, \tau_{n}+q \tau\right)}{\sigma_{u}\left(\tau_{n}+q \tau\right)}>\frac{y}{2} \theta^{-\eta^{\prime}}\right) \\
& \leq K_{1}\left(y \theta^{-\eta^{\prime}}\right)^{2 / \eta^{\prime}-1} e^{-\frac{\left(y \theta^{-\eta^{\prime}}\right)^{2}}{K}} .
\end{aligned}
$$

Consequently,

$$
\begin{aligned}
& \mathbb{P}\left(Z_{u}\left(s_{l}, \tau_{n}\right) \leq m(u)-\frac{\theta^{\eta}}{m(u)}, \sup _{(s, \tau) \in E_{l, n}(u)} Z_{u}(s, \tau)>m(u)\right) \\
& \leq \frac{K_{1}}{\sqrt{2 \pi} m(u)} e^{-\frac{m^{2}(u)}{2 \sigma_{u}^{2}\left(\tau_{n}\right)}} \int_{\theta^{\eta}}^{\infty} e^{2 y}\left(y \theta^{-\eta^{\prime}}\right)^{2 / \eta^{\prime}-1} e^{-\frac{\left(y \theta^{-\eta^{\prime}}\right)^{2}}{K}} d y \\
& \quad \leq \frac{K_{1}}{\sqrt{2 \pi} m(u)} e^{-\frac{m^{2}(u)}{2 \sigma_{u}^{2}\left(\tau_{n}\right)}} \theta^{\eta^{\prime}} \int_{\theta^{\eta-\eta^{\prime}}}^{\infty} e^{2 y \theta^{\eta^{\prime}}} y^{2 / \eta^{\prime}-1} e^{-\frac{y^{2}}{K}} d y \\
& \quad \leq \frac{K_{1}}{\sqrt{2 \pi} m(u)} e^{-\frac{m^{2}(u)}{2 \sigma_{u}^{2}\left(\tau_{n}\right)}} e^{-\frac{\theta^{2}\left(\eta-\eta^{\prime}\right)}{K_{2}}} .
\end{aligned}
$$

Using the above inequality and (3.3), we have that

$$
\begin{aligned}
& \mathbb{P}\left(\max _{\substack{0 \leq l \leq L \\
0 \leq|n| \leq N}} Z_{u}\left(s_{l}, \tau_{n}\right) \leq m(u)-\frac{\theta^{\eta}}{m(u)}, \sup _{(s, \tau) \in[0, T] \times J(u)} Z_{u}(s, \tau)>m(u)\right) \\
& \leq \sum_{0 \leq l \leq L,|n| \leq N} \mathbb{P}\left(Z_{u}\left(s_{l}, \tau_{n}\right) \leq m(u)-\frac{\theta^{\eta}}{m(u)}, \sup _{(s, \tau) \in E_{l, n}(u)} Z_{u}(s, \tau)>m(u)\right) \\
& \leq \sum_{0 \leq l \leq L,|n| \leq N} \frac{K_{1}}{\sqrt{2 \pi} m(u)} e^{-\frac{m^{2}(u)}{2 \sigma_{u}^{2}\left(\tau_{n}\right)}} e^{-\frac{\theta^{2}\left(\eta-\eta^{\prime}\right)}{K_{2}}} \\
& \leq L \frac{K_{1}}{\sqrt{2 \pi} m(u)} e^{-\frac{\theta^{2}\left(\eta-\eta^{\prime}\right)}{K_{2}}} \sum_{|n| \leq N} e^{-\frac{m^{2}(u)\left(1+B(n q)^{2} /(4 A)\right)}{2}} \\
& \quad \leq K_{1}\left(\frac{u}{m(u) \Delta(u)}\right)^{2} e^{-\frac{m^{2}(u)}{2}} \theta^{-2} e^{-\frac{\theta^{2}\left(\eta-\eta^{\prime}\right)}{K_{2}}}
\end{aligned}
$$




$$
\leq K_{1} \frac{u^{\gamma}}{m(u)} \Psi(m(u)) e^{-\frac{\theta^{2\left(\eta-\eta^{\prime}\right)}}{K_{2}}}
$$

This completes the proof.

Finally, by following the same arguments as in Dębicki and Liu (2016, Theorems 3.3) with the supremum functional substituted by its discrete counterpart, the maximum, we state the following result. Note that the asymptotic result below is a discrete version of (3.14) in Theorem 3.1.

Lemma 3.3. For any $T, \theta>0$, as $u \rightarrow \infty$,

$$
\mathbb{P}\left(\max _{\substack{0 \leq l \leq L \\ 0 \leq|n| \leq N}} Z_{u}\left(s_{l}, \tau_{n}\right)>m(u)\right)=\left(\mathcal{H}_{\eta_{\alpha_{\infty}}}^{\theta}\right)^{2} \sqrt{\frac{2 A \pi}{B}} \zeta_{\alpha_{\infty}} T \frac{u^{\gamma}}{m(u)} \Psi(m(u))(1+o(1)),
$$

where $\mathcal{H}_{\eta_{\alpha_{\infty}}}^{\theta}=\lim _{S \rightarrow \infty} S^{-1} \mathbb{E} \exp \left(\sup _{t \in \theta \mathbb{Z} \cap[0, S]}\left(\sqrt{2} \eta_{\alpha_{\infty}}(t)-\operatorname{Var}\left(\eta_{\alpha_{\infty}}(t)\right)\right)\right)$.

By the monotone convergence theorem, it follows that $\mathcal{H}_{\eta_{\alpha_{\infty}}}^{\theta} \rightarrow \mathcal{H}_{\eta_{\alpha \infty}}$ as $\theta \rightarrow 0$, since $H_{\eta_{\alpha \infty}}$ is a positive, finite constant and $\eta_{\alpha_{\infty}}$ has almost surely continuous sample paths. Consequently, when the discretization parameter $\theta$ decreases to zero so that the number of discretization points grows to infinity, we recover (3.14).

\section{Auxiliary Lemmas}

We begin with some auxiliary lemmas that are later needed in the proofs. The first lemma is (Leadbetter et al., 1983, Theorem 4.2.1).

Lemma 4.1 (Berman's inequality). Suppose $\xi_{1}, \ldots, \xi_{n}$ are standard normal variables with covariance matrix $\Lambda^{1}=\left(\Lambda_{i, j}^{1}\right)$ and $\eta_{1}, \ldots, \eta_{n}$ similarly with covariance matrix $\Lambda^{0}=\left(\Lambda_{i, j}^{0}\right)$. Let $\rho_{i, j}=\max \left(\left|\Lambda_{i, j}^{1}\right|,\left|\Lambda_{i, j}^{0}\right|\right)$ and let $u_{1}, \ldots, u_{n}$ be real numbers. Then,

$$
\begin{aligned}
\mathbb{P}\left(\bigcap_{j=1}^{n}\left\{\xi_{j} \leq u_{j}\right\}\right) & -\mathbb{P}\left(\bigcap_{j=1}^{n}\left\{\eta_{j} \leq u_{j}\right\}\right) \\
& \leq \frac{1}{2 \pi} \sum_{1 \leq i<j \leq n}\left(\Lambda_{i, j}^{1}-\Lambda_{i, j}^{0}\right)^{+}\left(1-\rho_{i, j}^{2}\right)^{-\frac{1}{2}} \exp \left(-\frac{u_{i}^{2}+u_{j}^{2}}{2\left(1+\rho_{i, j}\right)}\right) .
\end{aligned}
$$

The following lemma is a general form of the Borel-Cantelli lemma; cf. (Spitzer, 1964).

Lemma 4.2 (Borel-Cantelli lemma). Consider a sequence of event $\left\{E_{k}\right\}_{k=0}^{\infty}$. If

$$
\sum_{k=0}^{\infty} \mathbb{P}\left(E_{k}\right)<\infty
$$

then $\mathbb{P}\left(E_{n}\right.$ i.o. $)=0$. Whereas, if

$$
\sum_{k=0}^{\infty} \mathbb{P}\left(E_{k}\right)=\infty \quad \text { and } \quad \liminf _{n \rightarrow \infty} \frac{\sum_{1 \leq k \neq t \leq n} \mathbb{P}\left(E_{k} E_{t}\right)}{\left(\sum_{k=1}^{n} \mathbb{P}\left(E_{k}\right)\right)^{2}} \leq 1
$$

then $\mathbb{P}\left(E_{n}\right.$ i.o. $)=1$. 
Lemma 4.3. For any $\varepsilon \in(0,1)$, there exist positive constants $K$ and $\rho$ depending only on $\varepsilon, \alpha_{0}, \alpha_{\infty}$ and $p$ such that

$$
\begin{aligned}
& \mathbb{P}\left(\sup _{S<t \leq T} \frac{Q_{X}(t)}{f_{p}(t)} \leq 1\right) \\
& \leq \exp \left(-(1-\varepsilon) \int_{S+f_{p}(S)}^{T} \frac{1}{f_{p}(u)} \mathbb{P}\left(\sup _{t \in\left[0, f_{p}(u)\right]} Q_{X}(t)>f_{p}(u)\right) \mathrm{d} u\right)+K S^{-\rho}
\end{aligned}
$$

for any $T-f_{p}(S) \geq S \geq K$, with $f_{p}(T) / f_{p}(S) \leq \mathcal{C}$ and $\mathcal{C}$ being some universal positive constant.

Proof: Let $\varepsilon \in(0,1)$ be some positive constant. For the remainder of the proof let $K$ and $\rho$ be two positive constants depending only on $\varepsilon, \alpha_{0}, \alpha_{\infty}$ and $p$ that may differ from line to line. For any $k \geq 0$ put $s_{0}=S, y_{0}=f_{p}\left(s_{0}\right), t_{0}=s_{0}+y_{0}$, $x_{0}=f_{p}\left(t_{0}\right)$ and

$$
\begin{aligned}
& s_{k}=t_{k-1}+\varepsilon x_{k-1}, \quad y_{k}=f_{p}\left(s_{k}\right), \quad t_{k}=s_{k}+y_{k}, \quad x_{k}=f_{p}\left(t_{k}\right), \\
& I_{k}=\left(s_{k}, t_{k}\right], \quad \tilde{I}_{k}=\frac{I_{k}}{x_{k}}=\left(\tilde{s}_{k}, \tilde{t}_{k}\right], \quad\left|\tilde{I}_{k}\right|=\frac{y_{k}}{x_{k}} .
\end{aligned}
$$

From this construction, it is easy to see that the intervals $I_{k}$ are disjoint. Furthermore, $\delta\left(I_{k}, I_{k+1}\right)=\varepsilon x_{k}$, and $1-\varepsilon \leq y_{k} / x_{k} \leq 1$, for any $k \geq 0$ and sufficiently large $S$. Note that, for any $k \geq 0,\left|I_{k}\right| \geq f_{p}(S)$, therefore if $T(S, \varepsilon)$ is the smallest number of intervals $\left\{I_{k}\right\}$ needed to cover $[S, T]$, then $T(S, \varepsilon) \leq\left[(T-S) /\left(f_{p}(S)(1+\varepsilon)\right)\right]$. Moreover, since $f_{p}(T) / f_{p}(S)$ is bounded by the constant $\mathcal{C}>0$ not depending on $S$ and $\varepsilon$, it follows that, $x_{k} / x_{t} \leq \mathcal{C}$ for any $0 \leq t<k \leq T(S, \varepsilon)$.

Now let us introduce a discretization of the set $\tilde{I}_{k} \times J\left(x_{k}\right)$ as in Subsection 3.2. That is, for some $\theta=\frac{\Delta(S)}{S}$, define grid points

$$
\begin{aligned}
s_{k, l} & =\tilde{s}_{k}+l q_{k}, \quad 0 \leq l \leq L_{k}, \quad L_{k}=\left[(1-\varepsilon) / q_{k}\right], \quad q_{k}=\theta \frac{\Delta\left(x_{k}\right)}{x_{k}}, \\
\tau_{k, n} & =\tau\left(x_{k}\right)+n q_{k}, \quad 0 \leq|n| \leq N_{k}, \quad N_{k}=\left[\tau^{*}\left(x_{k}\right) / q_{k}\right] .
\end{aligned}
$$

Since $f_{p}$ is an increasing function, it easily follows that,

$$
\begin{aligned}
& \mathbb{P}\left(\sup _{S<t \leq T} \frac{Q_{X}(t)}{f_{p}(t)} \leq 1\right) \leq \mathbb{P}\left(\bigcap_{k=0}^{T(S, \varepsilon)}\left\{\sup _{t \in I_{k}} Q_{X}(t) \leq x_{k}\right\}\right) \\
& \leq \mathbb{P}\left(\bigcap_{k=0}^{T(S, \varepsilon)}\left\{\sup _{\substack{s \in I_{k} / x_{k} \\
\tau \in J\left(x_{k}\right)}} Z_{x_{k}}(s, \tau) \leq m\left(x_{k}\right)\right\}\right) \\
& \leq \mathbb{P}\left(\bigcap_{k=0}^{T(S, \varepsilon)}\left\{\max _{\substack{0 \leq l \leq L_{k} \\
0 \leq|n| \leq N_{k}}} Z_{x_{k}}\left(s_{k, l}, \tau_{k, n}\right) \leq m\left(x_{k}\right)\right\}\right) \\
& \leq \prod_{k=0}^{T(S, \varepsilon)} \mathbb{P}\left(\max _{\substack{0 \leq l \leq L_{k} \\
0 \leq|n| \leq N_{k}}} Z_{x_{k}}\left(s_{k, l}, \tau_{k, n}\right) \leq m\left(x_{k}\right)\right)+\sum_{0 \leq t<k \leq T(S, \varepsilon)} C_{k, t}=: P_{1}+P_{2},
\end{aligned}
$$


where the last inequality follows from Berman's inequality with

$$
\begin{aligned}
C_{k, t}= & \sum_{\substack{0 \leq l \leq L_{k} \\
0 \leq p \leq L_{t}}} \sum_{|n| \leq N_{k}} \frac{\left|r_{x_{k}, x_{t}}\left(s_{k, l}, \tau_{k, n}, s_{t, p}, \tau_{t, m}\right)\right|}{\sqrt{1-r_{x_{k}, x_{t}}^{2}\left(s_{k, l}, \tau_{k, n}, s_{t, p}, \tau_{t, m}\right)}} \\
& \times \exp \left(-\frac{\frac{1}{2}\left(m^{2}\left(x_{k}\right)+m^{2}\left(x_{t}\right)\right)}{1+\left|r_{x_{k}, x_{t}}\left(s_{k, l}, \tau_{k, n}, s_{t, p}, \tau_{t, m}\right)\right|}\right) .
\end{aligned}
$$

Estimation of $P_{1}$ :

Since for any $u$ the process $Z_{u}$ is stationary in the first variable, from Theorem 3.3 we have that, as $S \rightarrow \infty$ (noting that $\theta=\frac{\Delta(S)}{S} \rightarrow 0$ )

$$
\mathbb{P}\left(\max _{\substack{0 \leq l \leq L_{k} \\ 0 \leq|n| \leq N_{k}}} Z_{x_{k}}\left(s_{k, l}, \tau_{k, n}\right)>m\left(x_{k}\right)\right) \sim \mathbb{P}\left(\sup _{(s, \tau) \in \tilde{I}_{k} \times J\left(x_{k}\right)} Z_{x_{k}}(s, \tau)>m\left(x_{k}\right)\right)
$$

uniformly with respect to $0 \leq k \leq T(S, \varepsilon)$. Hence for any $\varepsilon \in(0,1)$, sufficiently large $S$ and small $\theta$,

$$
\begin{aligned}
P_{1} & \leq \exp \left(-\sum_{k=0}^{T(S, \varepsilon)} \mathbb{P}\left(\max _{\substack{0 \leq l \leq L_{k} \\
0 \leq|n| \leq N_{k}}} Z_{x_{k}}\left(s_{k, l}, \tau_{k, n}\right)>m\left(x_{k}\right)\right)\right) \\
& \leq \exp \left(-\left(1-\frac{\varepsilon}{8}\right) \sum_{k=0}^{T(S, \varepsilon)} \mathbb{P}\left(\sup _{(s, \tau) \in \tilde{I}_{k} \times J\left(x_{k}\right)} Z_{x_{k}}(s, \tau)>m\left(x_{k}\right)\right)\right)
\end{aligned}
$$

Then, by (3.1) combined with Theorem 3.1,

$$
\begin{aligned}
P_{1} & \leq \exp \left(-\left(1-\frac{\varepsilon}{4}\right) \sum_{k=0}^{T(S, \varepsilon)} \mathbb{P}\left(\sup _{s \in \tilde{I}_{k}} Z_{x_{k}}(s, \tau)>m\left(x_{k}\right)\right)\right) \\
& =\exp \left(-\left(1-\frac{\varepsilon}{4}\right) \sum_{k=0}^{T(S, \varepsilon)} \mathbb{P}\left(\sup _{t \in\left[0, \frac{y_{k}}{x_{k}} f_{p}\left(t_{k}\right)\right]} Q_{X}(t)>f_{p}\left(t_{k}\right)\right)\right) \\
& \leq \exp \left(-\left(1-\frac{\varepsilon}{2}\right) \sum_{k=0}^{T(S, \varepsilon)} \mathbb{P}\left(\sup _{t \in\left[0, f_{p}\left(t_{k}\right)\right]} Q_{X}(t)>f_{p}\left(t_{k}\right)\right) \frac{f_{p}\left(s_{k}\right)}{f_{p}\left(t_{k}\right)}\right) \\
& \leq \exp \left(-(1-\varepsilon) \int_{S+f_{p}(S)}^{T} \frac{1}{f_{p}(u)} \mathbb{P}\left(\sup _{t \in\left[0, f_{p}(u)\right]} Q_{X}(t)>f_{p}(u)\right) \mathrm{d} u\right)
\end{aligned}
$$

Estimation of $P_{2}$ :

For any $0 \leq t<k \leq T(S, \varepsilon), 0 \leq l \leq L_{k}, 0 \leq p \leq L_{t}$, we have

$$
\begin{aligned}
x_{k} s_{k, l}-x_{t} s_{t, p} & =\left(s_{k}+x_{k} l q_{k}\right)-\left(s_{t}+x_{t} p q_{t}\right) \\
& =\sum_{i=t}^{k-1}\left(y_{i}+\varepsilon x_{i}\right)+x_{k} l q_{k}-x_{t} p q_{t} \geq \sum_{i=t}^{k-1}\left(y_{i}+\varepsilon x_{i}\right)-y_{t} \\
& \geq \sum_{i=t+1}^{k-1} y_{i} .
\end{aligned}
$$


Recall that $\lambda=1-\alpha_{\infty}$. Hence we can find $s_{0}>2$ such that for $S$ sufficiently large, $k-t \geq 2 s_{0}, 0 \leq l \leq L_{k}, 0 \leq p \leq L_{t},|n| \leq N_{k}$ and $|m| \leq N_{t}$

$$
\frac{x_{k} \tau_{k, n}+x_{t} \tau_{t, m}}{\left|x_{k} s_{k, l}-x_{t} s_{t, p}\right|} \leq \frac{x_{k}\left(\tau_{k, n}+\tau_{t, m}\right)}{\sum_{i=t+1}^{k-1} y_{i}}<1 / 3
$$

which applied to (3.8) indicates that for $k-t \geq s_{0}$ and $S$ sufficiently large,

$$
\begin{aligned}
& r_{k, t}^{*}:=\sup _{\substack{0 \leq l \leq L_{k}, 0 \leq p \leq L_{t} \\
|n| \leq N_{k},|m| \leq N_{t}}}\left|r_{x_{k}, x_{t}}\left(s_{k, l}, \tau_{k, n}, s_{t, p}, \tau_{t, m}\right)\right| \\
& \leq 3^{2\left(1-\alpha_{\infty}\right)} \sup _{\substack{0 \leq l \leq L_{k}, 0 \leq p \leq L_{t} \\
|n| \leq N_{k},|m| \leq N_{t}}}\left|\frac{\sqrt{x_{k} \tau_{k, n} x_{t} \tau_{t, m}}}{\sum_{i=t+1}^{k-1} y_{i}}\right|^{2 \lambda} \\
& \leq 3^{2\left(1-\alpha_{\infty}\right)} \sup _{\substack{0 \leq l \leq L_{k}, 0 \leq p \leq L_{t} \\
|n| \leq N_{k},|m| \leq N_{t}}}\left|\frac{x_{k} \tau_{k, n}}{\sum_{i=t+1}^{k-1} y_{i}}\right|^{\lambda}\left|\frac{x_{t} \tau_{t, m}}{\sum_{i=t+1}^{k-1} y_{i}}\right|^{\lambda} \\
& \leq K\left|\frac{x_{t}}{\sum_{i=t+1}^{k-1} y_{i}}\right|^{\lambda} \leq K(k-t)^{-\lambda} \leq \frac{\lambda}{4} \text {. }
\end{aligned}
$$

For $1 \leq k-t \leq s_{0}$, it follows that $x_{k} \sim x_{t}, \tau_{k, l} \rightarrow \tau^{*}$ and $\tau_{t, p} \rightarrow \tau^{*}$ as $S \rightarrow \infty$, and $s_{k, l}-s_{t, p} \geq \epsilon x_{t} / x_{k}>\epsilon / 2$ for $S$ sufficiently large. Therefore, by (3.12) there exists a positive constant $\zeta \in(0,1)$ depending only on $\varepsilon$ such that for $S$ sufficiently large

$$
\sup _{1 \leq k-t \leq s_{0}} r_{k, t}^{*}=\sup _{1 \leq k-t \leq s_{0}} \sup _{\substack{0 \leq l \leq L_{k}, 0 \leq p \leq L_{t} \\|n| \leq N_{k},|m| \leq N_{t}}}\left|r_{x_{k}, x_{t}}\left(s_{k, l}, \tau_{k, n}, s_{t, p}, \tau_{t, m}\right)\right| \leq \zeta<1 .
$$

Finally, note that; c.f., (1.2),

$$
\begin{aligned}
N_{k} & \leq L_{k} \leq \frac{2(1-\varepsilon) x_{k}}{\theta \Delta\left(x_{k}\right)} \leq K x_{k}^{2 \gamma} \leq K\left(\log t_{k}\right)^{\frac{\gamma}{\left(1-\alpha_{\infty}\right)}} \\
\exp \left(-\frac{m^{2}\left(x_{k}\right)}{2}\right) & =\frac{\left(\log t_{k}\right)^{p-\frac{\gamma-1}{2(1-\alpha \infty)}}}{t_{k}}
\end{aligned}
$$

so that

$$
\begin{aligned}
& P_{2} \leq \frac{4}{\sqrt{1-\zeta^{2}}} \sum_{\substack{0 \leq t<k \leq T(S, \varepsilon)\\
}} L_{k} L_{t} N_{k} N_{t} r_{k, t}^{*} \exp \left(-\frac{m^{2}\left(x_{k}\right)+m^{2}\left(x_{t}\right)}{2\left(1+r_{k, t}^{*}\right)}\right) \\
& \leq K\left(\sum_{\substack{0<k-t \leq s_{0} \\
0 \leq t<k \leq T(S, \varepsilon)}}+\sum_{\substack{0 \leq t<k \leq T(S, \varepsilon) \\
0 \leq t>s_{0}}}\right)(\cdot) \\
& \leq K\left(\sum_{k=0}^{\infty} x_{k}^{8 \gamma} \exp \left(-\frac{m^{2}\left(x_{k}\right)}{1+\zeta}\right)\right. \\
&\left.+\sum_{\substack{k-t>s_{0} \\
0 \leq t<k \leq T(S, \varepsilon)}}\left(x_{k} x_{t}\right)^{4 \gamma}(k-t)^{-\lambda} \exp \left(-\frac{m^{2}\left(x_{k}\right)+m^{2}\left(x_{t}\right)}{2\left(1+\frac{\lambda}{4}\right)}\right)\right)
\end{aligned}
$$




$$
\begin{aligned}
& \leq K\left(\sum_{k=0}^{\infty} t_{k}^{-\frac{2}{1+\sqrt{\zeta}}}+\sum_{\substack{k-t>S_{0} \\
0 \leq t<k \leq T(S, \varepsilon)}} t_{k^{-\frac{1}{1+\frac{\lambda}{2}}}} t_{t}^{-\frac{1}{1+\frac{\lambda}{2}}}(k-t)^{-\lambda}\right) \\
& \leq K\left(\sum_{k=[S]}^{\infty} k^{-\frac{2}{1+\sqrt{\zeta}}}+\sum_{\substack{[S] \leq t<k \leq \infty \\
\leq K S^{-\rho}}} k^{\left.-\frac{1}{1+\frac{\lambda}{2}} t^{-\frac{1}{1+\frac{\lambda}{2}}}(k-t)^{-\lambda}\right)}\right. \\
& \leq
\end{aligned}
$$

where the last inequality follows from basic algebra.

Let $S>0$ be any fixed number, $a_{0}=S, y_{0}=f_{p}\left(a_{0}\right)$ and $b_{0}=a_{0}+y_{0}$. For $i>0$, define

$$
a_{i}=b_{i-1}, \quad y_{i}=f_{p}\left(a_{i}\right), \quad b_{i}=a_{i}+y_{i}, \quad M_{i}=\left(a_{i}, b_{i}\right], \quad \tilde{M}_{i}=\frac{M_{i}}{y_{i}}=\left(\tilde{a}_{i}, \tilde{b}_{i}\right] .
$$

From this construction, it is easy to see that the intervals $M_{i}$ are disjoint, $\cup_{j=0}^{i} M_{j}=$ $\left(S, b_{i}\right]$, and $\left|\tilde{M}_{i}\right|=1$. Now let us introduce a discretization of the set $\tilde{M}_{i} \times J\left(y_{i}\right)$ as in Subsection 3.2. That is, for $\theta=\frac{\Delta(S)}{S}$, define grid points

$$
\begin{aligned}
s_{i, l} & =\tilde{a}_{i}+l q_{i}, \quad 0 \leq l \leq L_{i}, \quad L_{i}=\left[1 / q_{i}\right], \quad q_{i}=\theta \frac{\Delta\left(y_{i}\right)}{y_{i}}, \\
\tau_{i, n} & =\tau\left(y_{i}\right)+n q_{i}, \quad 0 \leq|n| \leq N_{i}, \quad N_{i}=\left[\tau^{*}\left(y_{i}\right) / q_{i}\right] .
\end{aligned}
$$

With the above notation, we have the following lemma.

Lemma 4.4. For any $\varepsilon \in(0,1)$, there exists positive constants $K$ and $\rho$ depending only on $\varepsilon, \alpha_{0}, \alpha_{\infty}$ and $p$ such that, with $\theta_{i}=\left(m\left(y_{i}\right)\right)^{-4 / \hat{\alpha}}$, where $\hat{\alpha}=\min \left(\alpha_{0}, \alpha_{\infty}\right)$,

$$
\begin{aligned}
& \mathbb{P}\left(\bigcap_{i=0}^{\left[(T-S) / f_{p}(S)\right]}\left\{\max _{\substack{0 \leq l \leq L_{i} \\
0 \leq|n| \leq N_{i}}} Z_{y_{i}}\left(s_{i, l}, \tau_{i, n}\right) \leq m\left(y_{i}\right)-\frac{\theta_{i}^{\frac{\hat{\alpha}}{2}}}{m\left(y_{i}\right)}\right\}\right) \\
& \quad \geq \frac{1}{4} \exp \left(-(1+\varepsilon) \int_{S}^{T} \frac{1}{f_{p}(u)} \mathbb{P}\left(\sup _{t \in\left[0, f_{p}(u)\right]} Q_{X}(t)>f_{p}(u)\right) \mathrm{d} u\right)-K S^{-\rho},
\end{aligned}
$$

for any $T-f_{p}(S) \geq S \geq K$, with $f_{p}(T) / f_{p}(S) \leq \mathcal{C}$ and $\mathcal{C}$ being some universal positive constant.

Proof: Put

$$
\hat{m}\left(y_{i}\right)=m\left(y_{i}\right)-\frac{\theta_{i}^{\frac{\hat{\alpha}}{2}}}{m\left(y_{i}\right)}, \quad I=\left[(T-S) / f_{p}(S)\right] .
$$

Similarly as in the proof of Theorem 4.3 we find that Berman's inequality implies

$$
\begin{aligned}
& \mathbb{P}\left(\bigcap_{i=0}^{I}\left\{\max _{\substack{0 \leq l \leq L_{i} \\
0 \leq|n| \leq N_{i}}} Z_{y_{i}}\left(s_{i, l}, \tau_{i, n}\right) \leq m\left(y_{i}\right)-\frac{\theta_{i}^{\frac{\hat{\alpha}}{2}}}{m\left(y_{i}\right)}\right\}\right) \\
& \quad \geq \prod_{i=0}^{I} \mathbb{P}\left(\max _{\substack{0 \leq l \leq L_{i} \\
0 \leq|n| \leq N_{i}}} Z_{y_{i}}\left(s_{i, l}, \tau_{i, n}\right) \leq m\left(y_{i}\right)-\frac{\theta_{i}^{\frac{\hat{\alpha}}{2}}}{m\left(y_{i}\right)}\right)-\sum_{0 \leq i<j \leq I} D_{i, j}=: P_{1}^{\prime}+P_{2}^{\prime},
\end{aligned}
$$


where

$$
\begin{aligned}
D_{i, j}= & \frac{1}{2 \pi} \sum_{\substack{0 \leq l \leq L_{i} \\
0 \leq p \leq L_{j}}} \sum_{\substack{|n| \leq N_{i} \\
|m| \leq N_{j}}} \frac{\left(\tilde{r}_{y_{i}, y_{j}}\left(s_{i, l}, \tau_{i, n}, s_{j, p}, \tau_{j, m}\right)\right)^{+}}{\sqrt{1-\tilde{r}_{y_{i}, y_{j}}^{2}\left(s_{i, l}, \tau_{i, n}, s_{j, p}, \tau_{j, m}\right)}} \\
& \times \exp \left(-\frac{\frac{1}{2}\left(\hat{m}^{2}\left(y_{i}\right)+\hat{m}^{2}\left(y_{j}\right)\right)}{1+\left|\tilde{r}_{y_{i}, y_{j}}\left(s_{i, l}, \tau_{i, n}, s_{j, p}, \tau_{j, m}\right)\right|}\right)
\end{aligned}
$$

with

$$
\tilde{r}_{y_{i}, y_{j}}\left(s_{i, l}, \tau_{i, n}, s_{j, p}, \tau_{j, m}\right)=-r_{y_{i}, y_{j}}\left(s_{i, l}, \tau_{i, n}, s_{j, p}, \tau_{j, m}\right)
$$

Estimation of $P_{1}^{\prime}$ :

By Theorem 3.1 the correction term $\theta_{i}^{\hat{\alpha} / 2} / m\left(y_{i}\right)$ does not change the order of asymptotics of the tail of $Z_{y_{i}}$. Furthermore, the tail asymptotics of the supremum on the strip $(s, \tau) \in \tilde{M}_{i} \times J\left(y_{i}\right)$ are of the same order if $\tau \geq 0$. Hence, for every $\varepsilon>0$

$$
\begin{aligned}
P_{1}^{\prime} & \geq \frac{1}{4} \exp \left(-\sum_{i=0}^{I} \mathbb{P}\left(\max _{\substack{0 \leq l \leq L_{i} \\
0 \leq|n| \leq N_{i}}} Z_{y_{i}}\left(s_{i, l}, \tau_{i, n}\right)>\hat{m}\left(y_{i}\right)\right)\right) \\
& \geq \frac{1}{4} \exp \left(-\sum_{i=0}^{I} \mathbb{P}\left(\sup _{\substack{s \in \tilde{M}_{i} \\
\tau \in J\left(y_{i}\right)}} Z_{y_{i}}(s, \tau)>m\left(y_{i}\right)-\frac{\theta_{i}^{\frac{\hat{\alpha}}{2}}}{m\left(y_{i}\right)}\right)\right) \\
& \geq \frac{1}{4} \exp \left(-(1+\varepsilon) \sum_{i=0}^{I} \mathbb{P}\left(\sup _{s \in \tilde{M}_{i}} Z_{y_{i}}(s, \tau)>m\left(y_{i}\right)\right)\right) \\
& =\frac{1}{4} \exp \left(-(1+\varepsilon) \sum_{i=0}^{I} \mathbb{P}\left(\sup _{t \in\left[0, f_{p}\left(a_{i}\right)\right]} Q_{X}(t)>f_{p}\left(a_{i}\right)\right)\right) \\
& \left.\geq \frac{1}{4} \exp \left(-(1+\varepsilon) \int_{S}^{T} \frac{1}{f_{p}(u)} \mathbb{P}_{t \in\left[0, f_{p}(u)\right]} Q_{X}(t)>f_{p}(u)\right) \mathrm{d} u\right)
\end{aligned}
$$

provided that $S$ is sufficiently large along the same lines as the estimation of $P_{1}$ in Theorem 4.3.

\section{Estimation of $P_{2}^{\prime}$ :}

Clearly, for $j \geq i+2$, and any $0 \leq l \leq L_{i}, 0 \leq p \leq L_{j}$; c.f. (4.3),

$$
y_{j} s_{j, p}-y_{i} s_{i, l}=a_{j}+y_{j} p q_{j}-\left(a_{i}+y_{i} l q_{i}\right) \geq \sum_{k=i+1}^{j-1} y_{k} .
$$

Hence there exists $s_{0} \geq 2$ such that for $j-i \geq s_{0}, 0 \leq l \leq L_{i}, 0 \leq p \leq L_{j},|n| \leq N_{i}$, $|m| \leq N_{j}$ and $S$ sufficiently large

$$
\frac{y_{j} \tau_{j, m}+y_{i} \tau_{i, n}}{\left|y_{j} s_{j, p}-y_{i} s_{i, l}\right|} \leq \frac{y_{j}\left(\tau_{j, m}+\tau_{i, n}\right)}{\sum_{k=i+1}^{j-1} y_{k}} \leq \frac{1}{3} .
$$


Analogously as the derivation of (4.1), by (3.8) for $j-i \geq s_{0}$ and $S$ sufficiently large

$$
r_{i, j}^{*}:=\sup _{\substack{0 \leq l \leq L_{i}, 0 \leq p \leq L_{j} \\|n| \leq N_{i},|m| \leq N_{j}}}\left|\tilde{r}_{y_{i}, y_{j}}\left(s_{i, l}, \tau_{i, n}, s_{j, p}, \tau_{j, m}\right)\right| \leq K(k-t)^{-\lambda} \leq \frac{\lambda}{4},
$$

where $\lambda=1-\alpha_{\infty}$. For $1 \leq j-i \leq s_{0}$, it follows that $y_{i} \sim y_{j}, \tau_{i, n} \rightarrow \tau^{*}$ and $\tau_{j, m} \rightarrow \tau^{*}$ as $S \rightarrow \infty$, and $s_{i, l}-s_{j, p} \geq y_{i+1} / y_{j}>\frac{1}{2}$ for $2 \leq j-i \leq s_{0}$ and $S$ sufficiently large. Therefore, by (3.12) there exists a positive constant $\zeta_{1} \in(0,1)$ depending only on $\varepsilon$ such that for $S$ sufficiently large

$$
\sup _{2 \leq j-i \leq s_{0}} r_{i, j}^{*}=\sup _{2 \leq j-i \leq s_{0}} \sup _{\substack{0 \leq l \leq L_{i}, 0 \leq p \leq L_{j} \\|n| \leq N_{i},|m| \leq N_{j}}}\left|r_{y_{i}, y_{j}}\left(s_{i, l}, \tau_{i, n}, s_{j, p}, \tau_{j, m}\right)\right| \leq \zeta_{1} .
$$

Moreover, by (3.11) there exist positive constants $\delta \in(0,1)$ and $c_{\delta} \in\left(0, \frac{1}{2}\right)$, $M<1$, such that, for sufficiently large $S$,

$$
\inf _{\left|y_{i}-y_{j}\right|<c_{\delta},\left|\tau-\tau^{*}\right|,\left|\tau^{\prime}-\tau^{*}\right| \leq M} r_{y_{i}, y_{j}}\left(s, \tau, s^{\prime}, \tau^{\prime}\right)>\frac{\delta}{2} .
$$

Hence for sufficiently large $S$ and $0 \leq l \leq L_{i}, 0 \leq p \leq L_{j},|n| \leq N_{i},|m| \leq N_{j}$

$$
\left(\tilde{r}_{y_{i}, y_{j}}\left(s_{i, l}, \tau_{i, n}, s_{j, p}, \tau_{j, m}\right)\right)^{+}=0, \quad \text { if } \quad j=i+1, \quad\left|s_{i, l}-s_{j, p}\right| \leq c_{\delta} .
$$

By (3.12) there exits $\zeta_{2} \in(0,1)$ such that for $S$ sufficiently large and $0 \leq l \leq L_{i}$, $0 \leq p \leq L_{j},|n| \leq N_{i},|m| \leq N_{j}$

$$
\left|\tilde{r}_{y_{i}, y_{j}}\left(s_{i, l}, \tau_{i, n}, s_{j, p}, \tau_{j, m}\right)\right| \leq \zeta_{2}, \quad \text { if } \quad j=i+1, \quad\left|s_{i, l}-s_{j, p}\right| \geq c_{\delta} .
$$

Let $\zeta=\max \left(\zeta_{1}, \zeta_{2}\right)$. Therefore, by (4.2)-(4.7) we obtain

$$
\begin{aligned}
P_{2}^{\prime} \leq & \sum_{\substack{0 \leq i \leq I-1 \\
1 \leq j-i \leq s_{0} 0}} \sum_{\substack{0 \leq l \leq L_{i} \\
0 \leq p \leq L_{j}}} \sum_{\substack{|n| \leq N_{i} \\
|m| \leq N_{j}}} \frac{1}{\sqrt{1-\zeta}} \exp \left(-\frac{\frac{1}{2}\left(\hat{m}^{2}\left(y_{i}\right)+\hat{m}^{2}\left(y_{j}\right)\right)}{1+\zeta}\right) \\
& +\sum_{\substack{0 \leq i \leq I-2 \\
i+s_{0} \leq j \leq I}} \sum_{\substack{0 \leq l \leq L_{i} \\
0 \leq p \leq L_{j}}} \sum_{\substack{|n| \leq N_{i} \\
|m| \leq N_{j}}} \frac{r_{i, j}^{*}}{\sqrt{1-r_{i, j}^{*}}} \exp \left(-\frac{\frac{1}{2}\left(\hat{m}^{2}\left(y_{i}\right)+\hat{m}^{2}\left(y_{j}\right)\right)}{1+r_{i, j}^{*}}\right) .
\end{aligned}
$$

Completely similar to the estimation of $P_{2}$ in the proof of Theorem 4.3, we can arrive that there exist positive constants $K$ and $\rho$ such that, for sufficiently large $S$,

$$
P_{2}^{\prime} \leq K S^{-\rho} \text {. }
$$

The next lemma is a straightforward modification of (Watanabe, 1970, Lemma 3.1 and Lemma 4.1), see also (Qualls and Watanabe, 1971, Lemma 1.4).

Lemma 4.5. It is enough to proof Theorem 1.1 for any nondecreasing function $f$ such that,

$$
\overleftarrow{m}(\sqrt{\log t}) \leq f(t) \leq \overleftarrow{m}(\sqrt{3 \log t})
$$

for all $t \geq T$, and $T$ large enough. 


\section{Proof of the main results}

Proof of Theorem 1.1: Note that the case $\mathscr{I}_{f}<\infty$ is straightforward and does not need any additional knowledge on the process $Q_{X}$ apart from the property of stationarity. Indeed, consider the sequence of intervals $M_{i}$ as in Theorem 4.4. Then, for any $\varepsilon>0$ and sufficiently large $T$,

$$
\sum_{k=[T]+1}^{\infty} \mathbb{P}\left(\sup _{t \in M_{k}} Q_{X}(t)>f\left(a_{k}\right)\right)=\sum_{k=[T]}^{\infty} \mathbb{P}\left(\sup _{t \in\left[0, f\left(b_{k}\right)\right]} Q_{X}(t)>f\left(b_{k}\right)\right) \leq \mathscr{I}_{f}<\infty
$$

and the Borel-Cantelli lemma completes this part of the proof since $f$ is an increasing function.

Now let $f$ be an increasing function such that $\mathscr{I}_{f} \equiv \infty$. With the same notation as in Theorem 4.3 with $f$ instead of $f_{p}$, we find that, for any $S, \varepsilon, \theta>0$,

$$
\begin{aligned}
\mathbb{P}\left(Q_{X}(s)>f(s) \text { i.o. }\right) & \geq \mathbb{P}\left(\left\{\sup _{t \in I_{k}} Q_{X}(t)>f\left(t_{k}\right)\right\} \text { i.o. }\right) \\
& \geq \mathbb{P}\left(\left\{\max _{\substack{0 \leq l \leq L k \\
0 \leq|n| \leq N_{k}}} Z_{x_{k}}\left(s_{k, l}, \tau_{k, n}\right)>m\left(x_{k}\right)\right\} \text { i.o. }\right) .
\end{aligned}
$$

Let

$$
E_{k}=\left\{\max _{\substack{0 \leq l \leq L_{k} \\ 0 \leq|n| \leq N_{k}}} Z_{x_{k}}\left(s_{k, l}, \tau_{k, n}\right) \leq m\left(x_{k}\right)\right\}
$$

For sufficiently large $S$ and sufficiently small $\theta$; c.f., estimation of $P_{1}$, we get

$$
\sum_{k=0}^{\infty} \mathbb{P}\left(E_{k}^{c}\right) \geq(1-\varepsilon) \int_{S+f(S)}^{\infty} \frac{1}{f(u)} \mathbb{P}\left(\sup _{t \in[0, f(u)]} Q_{X}(t)>f(u)\right) \mathrm{d} u=\infty .
$$

Note that

$$
1-\mathbb{P}\left(E_{i}^{c} \quad \text { i.o. }\right)=\lim _{m \rightarrow \infty} \prod_{k=m}^{\infty} \mathbb{P}\left(E_{k}\right)+\lim _{m \rightarrow \infty}\left(\mathbb{P}\left(\bigcap_{k=m}^{\infty} E_{k}\right)-\prod_{k=m}^{\infty} \mathbb{P}\left(E_{k}\right)\right) .
$$

The first limit is zero as a consequence of (5.1), and the second limit will be zero because of the asymptotic independence of the events $E_{k}$. Indeed, there exist positive constants $K$ and $\rho$, depending only on $\alpha_{0}, \alpha_{\infty}, \varepsilon, \lambda$, such that for any $n>m$,

$$
A_{m, n}=\left|\mathbb{P}\left(\bigcap_{k=m}^{n} E_{k}\right)-\prod_{k=m}^{n} \mathbb{P}\left(E_{k}\right)\right| \leq K(S+m)^{-\rho}
$$

by the same calculations as in the estimate of $P_{2}$ in Theorem 4.3 after realizing that, by Theorem 4.5, we might restrict ourselves to the case when (4.8) holds. Therefore $\mathbb{P}\left(E_{i}^{c}\right.$ i.o. $)=1$, which finishes the proof.

Proof of Theorem 1.3:

Let $\xi_{p} \equiv \xi_{f_{p}}$ for short.

Step 1. Let $p>1$, then, for every $\varepsilon \in\left(0, \frac{1}{4}\right)$,

$$
\liminf _{t \rightarrow \infty} \frac{\xi_{p}(t)-t}{h_{p}(t)} \geq-(1+2 \varepsilon) \quad \text { a.s. }
$$


Proof: Let $\left\{T_{k}: k \geq 1\right\}$ be a sequence such that $T_{k} \rightarrow \infty$, as $k \rightarrow \infty$. Put $S_{k}=T_{k}-(1+2 \varepsilon) h_{p}\left(T_{k}\right)$. Since $h_{p}(t)=O\left(t \log ^{1-p} t \log _{2} t\right)$, then, for $p>1$, $S_{k} \sim T_{k}$, as $k \rightarrow \infty$, and from Theorem 4.3 it follows that

$$
\begin{aligned}
& \mathbb{P}\left(\frac{\xi_{p}\left(T_{k}\right)-T_{k}}{h_{p}\left(T_{k}\right)} \leq-(1+2 \varepsilon)^{2}\right)=\mathbb{P}\left(\xi_{p}\left(T_{k}\right) \leq S_{k}\right)=\mathbb{P}\left(\sup _{S_{k}<t \leq T_{k}} \frac{Q_{X}(t)}{f_{p}(t)}<1\right) \\
& \quad \leq \exp \left(-(1-\varepsilon) \int_{S_{k}+f_{p}\left(S_{k}\right)}^{T_{k}} \frac{1}{f_{p}(u)} \mathbb{P}\left(\sup _{t \in\left[0, f_{p}(u)\right]} Q_{X}(t)>f_{p}(u)\right) \mathrm{d} u\right)+2 K T_{k}^{-\rho} .
\end{aligned}
$$

Moreover, as $k \rightarrow \infty$,

$$
\begin{aligned}
& \int_{S_{k}+f_{p}\left(S_{k}\right)}^{T_{k}} \frac{1}{f_{p}(u)} \mathbb{P}\left(\sup _{t \in\left[0, f_{p}(u)\right]} Q_{X}(t)>f_{p}(u)\right) \mathrm{d} u \\
& \sim(1+2 \varepsilon) h_{p}\left(T_{k}\right) \frac{1}{f_{p}\left(T_{k}\right)} \mathbb{P}\left(\sup _{t \in\left[0, f_{p}\left(T_{k}\right)\right]} Q_{X}(t)>f_{p}\left(T_{k}\right)\right)=(1+2 \varepsilon) p \log _{2} T_{k} .
\end{aligned}
$$

Now take $T_{k}=\exp \left(k^{1 / p}\right)$. Then,

$$
\sum_{k=1}^{\infty} \mathbb{P}\left(\xi_{p}\left(T_{k}\right) \leq S_{k}\right) \leq 2 K \sum_{k=1}^{\infty} k^{-(1+\varepsilon / 2)}<\infty .
$$

Hence by the Borel-Cantelli lemma,

$$
\liminf _{k \rightarrow \infty} \frac{\xi_{p}\left(T_{k}\right)-T_{k}}{h_{p}\left(T_{k}\right)} \geq-(1+2 \varepsilon) \quad \text { a.s.. }
$$

Since $\xi_{p}(t)$ is a non-decreasing random function of $t$, for every $T_{k} \leq t \leq T_{k+1}$, we have

$$
\frac{\xi_{p}(t)-t}{h_{p}(t)} \geq \frac{\xi_{p}\left(T_{k}\right)-T_{k}}{h_{p}\left(T_{k}\right)}-\frac{T_{k+1}-T_{k}}{h_{p}\left(T_{k}\right)} .
$$

For $p>1$ elementary calculus implies

$$
\lim _{k \rightarrow \infty} \frac{T_{k+1}-T_{k}}{h_{p}\left(T_{k}\right)}=0,
$$

so that

$$
\liminf _{t \rightarrow \infty} \frac{\xi_{p}(t)-t}{h_{p}(t)} \geq \liminf _{k \rightarrow \infty} \frac{\xi_{p}\left(T_{k}\right)-T_{k}}{h_{p}\left(T_{k}\right)} \text { a.s., }
$$

which finishes the proof of this step.

Step 2. Let $p>1$, then, for every $\varepsilon \in(0,1)$,

$$
\liminf _{t \rightarrow \infty} \frac{\xi_{p}(t)-t}{h_{p}(t)} \leq-(1-\varepsilon) \quad \text { a.s. }
$$

Proof: As in the proof of the lower bound, put

$$
T_{k}=\exp \left(k^{\left(1+\varepsilon^{2}\right) / p}\right), \quad S_{k}=T_{k}-(1-\varepsilon) h_{p}\left(T_{k}\right), \quad k \geq 1 .
$$

Let

$$
B_{k}=\left\{\xi_{p}\left(T_{k}\right) \leq S_{k}\right\}=\left\{\sup _{S_{k}<t \leq T_{k}} \frac{Q_{X}(t)}{f_{p}(t)}<1\right\} .
$$


It suffices to show $\mathbb{P}\left(B_{n}\right.$ i.o. $)=1$, that is

$$
\lim _{m \rightarrow \infty} \mathbb{P}\left(\bigcup_{k=m}^{\infty} B_{k}\right)=1
$$

Let

$$
\begin{gathered}
a_{0}^{k}=S_{k}, \quad y_{0}^{k}=f_{p}\left(a_{0}^{k}\right), \quad b_{0}^{k}=a_{0}^{k}+y_{0}^{k}, \\
a_{i}^{k}=b_{i-1}^{k}, \quad y_{i}^{k}=f_{p}\left(a_{i}^{k}\right), \quad b_{i}^{k}=a_{i}^{k}+y_{i}^{k}, \quad M_{i}^{k}=\left(a_{i}^{k}, b_{i}^{k}\right], \quad \tilde{M}_{i}^{k}=\frac{M_{i}^{k}}{y_{i}^{k}}=\left(\tilde{a}_{i}^{k}, \tilde{b}_{i}^{k}\right] .
\end{gathered}
$$

Define $J_{k}$ to be the biggest number such that $b_{J_{k}-1}^{k} \leq T_{k}$ and $b_{J_{k}}^{k}>T_{k}$. Note that $J_{k} \leq\left[\left(T_{k}-S_{k}\right) / f_{p}\left(S_{k}\right)\right]$.

Since $f_{p}$ is an increasing function,

$$
\begin{aligned}
B_{k} \supset \bigcap_{i=0}^{J_{k}}\left\{\sup _{t \in M_{i}^{k}} \frac{Q_{X}(t)}{f_{p}(t)}<1\right\} & \supset \bigcap_{i=0}^{J_{k}}\left\{\sup _{t \in M_{i}^{k}} Q_{X}(t)<y_{i}^{k}\right\} \\
& =\bigcap_{i=0}^{J_{k}}\left\{\sup _{\substack{s \in \tilde{M}_{i}^{k} \\
\tau \geq 0}} Z_{y_{i}^{k}}(s, \tau)<m\left(y_{i}^{k}\right)\right\}
\end{aligned}
$$

Analogously to (4.4), define a discretization of the set $\tilde{M}_{i}^{k} \times J\left(y_{i}^{k}\right)$ as follows

$$
\begin{aligned}
& s_{i, l}^{k}=\tilde{a}_{i}^{k}+l q_{i}^{k}, 0 \leq l \leq L_{i}^{k}, L_{i}^{k}=\left[1 / q_{i}^{k}\right], q_{i}^{k}=\theta_{i}^{k} \frac{\Delta\left(y_{i}^{k}\right)}{y_{i}^{k}}, \theta_{i}^{k}=\left(m\left(y_{i}^{k}\right)\right)^{-4 / \hat{\alpha}}, \\
& \tau_{i, n}^{k}=\tau\left(y_{i}^{k}\right)+n q_{i}^{k}, 0 \leq|n| \leq N_{i}^{k}, N_{i}^{k}=\left[\tau^{*}\left(y_{i}^{k}\right) / q_{i}^{k}\right] .
\end{aligned}
$$

Recall that $\hat{\alpha}=\min \left(\alpha_{0}, \alpha_{\infty}\right)$ and let

$$
A_{k}=\bigcap_{i=0}^{J_{k}}\left\{\max _{\substack{0 \leq l \leq L_{i}^{k} \\ 0 \leq|n| \leq N_{i}^{k}}} Z_{y_{i}^{k}}\left(s_{i, l}^{k}, \tau_{i, n}^{k}\right) \leq m\left(y_{i}^{k}\right)-\frac{\left(\theta_{i}^{k}\right)^{\frac{\hat{\alpha}}{2}}}{m\left(y_{i}^{k}\right)}\right\} .
$$

Observe that

$$
\mathbb{P}\left(\bigcup_{k=m}^{\infty} A_{k}\right) \leq \mathbb{P}\left(\bigcup_{k=m}^{\infty} B_{k}\right)+\sum_{k=m}^{\infty} \mathbb{P}\left(A_{k} \cap B_{k}^{c}\right)
$$

Furthermore,

$$
\begin{aligned}
& \sum_{k=m}^{\infty} \mathbb{P}\left(A_{k} \cap B_{k}^{c}\right) \\
& \leq \sum_{k=m}^{\infty} \sum_{i=0}^{J_{k}} \mathbb{P}\left(\max _{\substack{0 \leq l \leq L_{i}^{k} \\
0 \leq|n| \leq N_{i}^{k}}} Z_{y_{i}^{k}}\left(s_{i, l}^{k}, \tau_{i, n}^{k}\right) \leq m\left(y_{i}^{k}\right)-\frac{\left(\theta_{i}^{k}\right)^{\frac{\hat{\alpha}}{2}}}{m\left(y_{i}^{k}\right)}, \sup _{\substack{s \in \tilde{M}_{i}^{k} \\
\tau \geq 0}} Z_{y_{i}^{k}}(s, \tau) \geq m\left(y_{i}^{k}\right)\right) \\
& \leq \sum_{k=m}^{\infty} \sum_{i=0}^{J_{k}} \mathbb{P}\left(\max _{\substack{0 \leq l \leq L_{i}^{k} \\
0 \leq|n| \leq N_{i}^{k}}} Z_{y_{i}^{k}}\left(s_{i, l}^{k}, \tau_{i, n}^{k}\right) \leq m\left(y_{i}^{k}\right)-\frac{\left(\theta_{i}^{k}\right)^{\frac{\hat{\alpha}}{2}}}{m\left(y_{i}^{k}\right)}, \sup _{\substack{\left.\operatorname{sum}_{i \in J\left(\tilde{M}_{i}^{k}\right)} \\
\tau \in y_{i}^{k}\right)}} Z_{y_{i}^{k}}(s, \tau) \geq m\left(y_{i}^{k}\right)\right.
\end{aligned}
$$




$$
+\sum_{k=m}^{\infty} \sum_{i=0}^{J_{k}} \mathbb{P}\left(\sup _{\substack{s \in \tilde{M}_{i}^{k} \\ \tau \notin J\left(y_{i}^{k}\right)}} Z_{y_{i}^{k}}(s, \tau) \geq m\left(y_{i}^{k}\right)\right) .
$$

By Theorem 3.1 and Theorem 3.2, for sufficiently large $m$ and some $K_{1}, K_{2}>0$, the first sum is bounded from above by

$$
\begin{aligned}
\sum_{k=m}^{\infty} \sum_{i=0}^{J_{k}} & K_{1} \frac{\left(y_{i}^{k}\right)^{\gamma}}{m\left(y_{i}^{k}\right)} \Psi\left(m\left(y_{i}^{k}\right)\right) e^{-\left(m\left(y_{i}^{k}\right)\right)^{3} / K_{2}} \\
& \leq \sum_{k=m}^{\infty} \sum_{i=0}^{J_{k}} K_{1} \mathbb{P}\left(\sup _{(s, \tau) \in[0,1] \times \mathbb{R}_{+}} Z_{y_{i}^{k}}(s, \tau)>m\left(y_{i}^{k}\right)\right) e^{-\left(m\left(y_{i}^{k}\right)\right)^{3} / K_{2}} \\
& \leq \sum_{k=m}^{\infty} \sum_{i=0}^{J_{k}} K_{1} \mathbb{P}\left(\sup _{t \in\left[0, f_{p}\left(a_{i}^{k}\right)\right]} Q_{X}(t)>f_{p}\left(a_{i}^{k}\right)\right) e^{-\left(\log a_{i}^{k}\right)^{3 / 2} / K_{2}} \\
& \leq K \int_{m}^{\infty} \frac{\psi\left(f_{p}(x)\right)}{f_{p}(x)} e^{-\log ^{3 / 2}(x) / K_{2}} \mathrm{~d} x<\infty .
\end{aligned}
$$

Note that by (3.13), for sufficiently large $m$, the term in (5.5) is bounded from above by

$$
\begin{aligned}
& K \sum_{k=m}^{\infty} \sum_{i=0}^{J_{k}} \frac{\left(y_{i}^{k}\right)^{\gamma}}{m\left(y_{i}^{k}\right)} \Psi\left(m\left(y_{i}^{k}\right)\right) \exp \left(-\frac{b}{4} \log ^{2} m\left(y_{i}^{k}\right)\right) \\
& \leq K \int_{m}^{\infty} \frac{\psi\left(f_{p}(x)\right)}{f_{p}(x)} e^{\left.-\frac{b}{4}\left(\frac{1}{2} \log _{2} x\right)\right)^{2}} \mathrm{~d} x \\
& <\infty
\end{aligned}
$$

Therefore

$$
\lim _{m \rightarrow \infty} \sum_{k=m}^{\infty} \mathbb{P}\left(A_{k} \cap B_{k}^{c}\right)=0
$$

and

$$
\lim _{m \rightarrow \infty} \mathbb{P}\left(\bigcup_{k=m}^{\infty} B_{k}\right) \geq \lim _{m \rightarrow \infty} \mathbb{P}\left(\bigcup_{k=m}^{\infty} A_{k}\right) .
$$

To finish the proof of (5.4), we only need to show that

$$
\mathbb{P}\left(A_{n} \text { i.o. }\right)=1 .
$$

Similarly to (5.2), we have

$$
\int_{S_{k}}^{T_{k}} \frac{1}{f_{p}(u)} \mathbb{P}\left(\sup _{t \in\left[0, f_{p}(u)\right]} Q_{X}(t)>f_{p}(u)\right) \mathrm{d} u \sim(1-\varepsilon) p \log _{2} T_{k} .
$$

Now from Theorem 4.4 it follows that

$$
\mathbb{P}\left(A_{k}\right) \geq \frac{1}{4} \exp \left(-\left(1-\varepsilon^{2}\right) p \log _{2} T_{k}\right)-K S_{k}^{-\rho} \geq \frac{1}{8} k^{-\left(1-\varepsilon^{4}\right)},
$$

for every $k$ sufficiently large. Hence,

$$
\sum_{k=1}^{\infty} \mathbb{P}\left(A_{k}\right)=\infty
$$


Applying Berman's inequality, we get for $t<k$

$$
\mathbb{P}\left(A_{k} A_{t}\right) \leq \mathbb{P}\left(A_{k}\right) \mathbb{P}\left(A_{t}\right)+Q_{k, t},
$$

where,

$$
\begin{aligned}
Q_{k, t}=\sum_{\substack{0 \leq i \leq J_{k} \\
0 \leq j \leq J_{t}}} \sum_{0 \leq l \leq L_{i}^{k}} \sum_{\substack{0 \leq p \leq L_{j}^{t}\left|\leq N_{i}^{k}\right| \leq N_{j}^{t} \\
0}} \frac{\left|r_{y_{i}^{k}, y_{j}^{t}}\left(s_{i, l}^{k}, \tau_{i, n}^{k}, s_{j, p}^{t}, \tau_{j, m}^{t}\right)\right|}{\sqrt{1-r_{y_{i}^{k}, y_{j}^{t}}^{2}\left(s_{i, l}^{k}, \tau_{i, n}^{k}, s_{j, p}^{t}, \tau_{j, m}^{t}\right)}} \\
\quad \times \exp \left(-\frac{\left(m\left(y_{i}^{k}\right)-\left(m\left(y_{i}^{k}\right)\right)^{-3}\right)^{2}+\left(m\left(y_{j}^{t}\right)-\left(m\left(y_{j}^{t}\right)\right)^{-3}\right)^{2}}{2\left(1+\left|r_{y_{i}^{k}, y_{j}^{t}}\left(s_{i, l}^{k}, \tau_{i, n}^{k}, s_{j, p}^{t}, \tau_{j, m}^{t}\right)\right|\right)}\right) .
\end{aligned}
$$

For any $0 \leq i \leq J_{k}, 0 \leq j \leq J_{t}, 0 \leq l \leq L_{i}^{k}, 0 \leq p \leq L_{j}^{t}$, and $t<k$,

$y_{i}^{k} s_{i, l}^{k}-y_{j}^{t} s_{j, p}^{t}=a_{i}^{k}+y_{i}^{k} l q_{i}^{k}-\left(a_{j}^{t}+y_{j}^{t} p q_{j}^{t}\right) \geq S_{k}-T_{t} \geq S_{k}-T_{k-1} \geq \frac{1}{2}\left(T_{k}-T_{k-1}\right)$, where the last inequality holds for $k$ large enough since it is easy to see that

$$
\frac{S_{k+1}-T_{k}}{T_{k+1}-T_{k}} \sim 1, \quad \text { as } k \rightarrow \infty .
$$

Thus, sufficiently large $k$ and every $0 \leq t<k$, and a generic constant $K>0$, similarly to (4.1) we have,

$$
\sup _{\substack{0 \leq i \leq J_{k} \\ 0 \leq j \leq J_{t} \\ 0 \leq l \leq L_{i}^{k}, 0 \leq p \leq L_{j}^{t} \\|n| \leq N_{i}^{k},|m| \leq N_{j}^{t}}}\left|r_{y_{i}^{k}, y_{j}^{t}}\left(s_{i, l}^{k}, \tau_{n}^{k}, s_{j, p}^{t}, \tau_{j, m}^{t}\right)\right| \leq K\left(T_{k}-T_{k-1}\right)^{-\lambda / 2} \leq \frac{\min (1, \lambda)}{32} .
$$

Therefore, for some generic constant $K$ not depending on $k$ and $t$ which may vary between lines, for every $t<k$ sufficiently large,

$$
\begin{aligned}
Q_{k, t} \leq K \sum_{\substack{0 \leq i \leq J_{k} \\
0 \leq j \leq J_{t}}} L_{i}^{k} L_{j}^{t} N_{i}^{k} N_{j}^{t}\left(T_{k}-T_{k-1}\right)^{-\lambda / 2} \exp \left(-\frac{\left(m\left(y_{i}^{k}\right)\right)^{2}+\left(m\left(y_{j}^{t}\right)\right)^{2}}{2\left(1+\frac{\lambda}{16}\right)}\right) \\
\leq K\left(T_{k}-T_{k-1}\right)^{-\lambda / 2}\left(L_{J_{k}}^{k} L_{J_{t}}^{t}\right)^{2} \\
\quad \times \sum_{\substack{0 \leq i \leq J_{k} \\
0 \leq j \leq J_{t}}}\left(a_{i}^{k} \log ^{\frac{\gamma-1}{2(1-\alpha \infty)}-p} a_{i}^{k}\right)^{-\frac{1}{1+\frac{\lambda}{16}}}\left(a_{j}^{t} \log ^{\frac{\gamma-1}{2(1-\alpha \infty)}-p} a_{j}^{t}\right)^{-\frac{1}{1+\frac{\lambda}{16}}} \\
\leq K\left(T_{k}-T_{k-1}\right)^{-\lambda / 2}\left(\log T_{k}\right)^{v}\left(T_{k}\right)^{\frac{\frac{\lambda}{8}}{1+\frac{\lambda}{8}}}\left(T_{t}\right)^{\frac{\lambda}{8}} \frac{\lambda}{1+\frac{\lambda}{8}} \\
\leq K T_{k}^{-\lambda / 8} \leq K \exp \left(-\lambda k^{\left.\left(1+\varepsilon^{2}\right) / p / 8\right),}\right.
\end{aligned}
$$

with $v>0$ a fixed constant. Hence we have,

$$
\sum_{0 \leq t<k<\infty} Q_{k, t}<\infty
$$

Now (5.6) follows from (5.7)-(5.9) and the general form of the Borel-Cantelli lemma. 
Step 3. If $p \in(0,1]$, then, for every $\varepsilon \in\left(0, \frac{1}{4}\right)$,

$$
\liminf _{t \rightarrow \infty} \frac{\log \left(\xi_{p}(t) / t\right)}{h_{p}(t) / t} \geq-(1+2 \varepsilon) \quad \text { a.s. }
$$

and

$$
\liminf _{t \rightarrow \infty} \frac{\log \left(\xi_{p}(t) / t\right)}{h_{p}(t) / t} \leq-(1-\varepsilon) \quad \text { a.s. }
$$

Proof: Put

$$
T_{k}=\exp (k), \quad S_{k}=T_{k} \exp \left(-(1+2 \varepsilon) h_{p}\left(T_{k}\right) / T_{k}\right) .
$$

Proceeding the same as in the proof of (5.3), one can obtain that

$$
\liminf _{k \rightarrow \infty} \frac{\log \left(\xi_{p}\left(T_{k}\right) / T_{k}\right)}{h_{p}\left(T_{k}\right) / T_{k}} \geq-(1+2 \varepsilon) \quad \text { a.s. }
$$

On the other hand it is clear that

$$
\liminf _{t \rightarrow \infty} \frac{\log \left(\xi_{p}(t) / t\right)}{h_{p}(t) / t}=\liminf _{k \rightarrow \infty} \frac{\log \left(\xi_{p}\left(T_{k}\right) / T_{k}\right)}{h_{p}\left(T_{k}\right) / T_{k}} \quad \text { a.s. }
$$

since

$$
\liminf _{k \rightarrow \infty} \frac{\log \left(T_{k} / T_{k+1}\right)}{h_{p}\left(T_{k}\right) / T_{k}}=0 .
$$

This proves (5.10).

Let

$$
T_{k}=\exp \left(k^{1+\varepsilon^{2}}\right), \quad S_{k}=T_{k} \exp \left(-(1-\varepsilon) h_{p}\left(T_{k}\right) / T_{k}\right) .
$$

Noting that

$$
\frac{S_{k+1}-T_{k}}{S_{k+1}} \sim 1 \quad \text { as } k \rightarrow \infty,
$$

along the same line as in the proof of (5.4), we also have

$$
\liminf _{k \rightarrow \infty} \frac{\log \left(\xi_{p}\left(T_{k}\right) / T_{k}\right)}{h_{p}\left(T_{k}\right) / T_{k}} \leq-(1-\varepsilon) \quad \text { a.s., }
$$

which proves (5.11).

\section{Appendix}

Proof of (3.10). Let $g_{1}(t)=g\left(\tau^{*} t\right)$. Then it suffices to prove the claim in (3.10) for

$$
g_{1}(t)=\frac{|1+t|^{2 \alpha_{\infty}}+|1-t|^{2 \alpha_{\infty}}-2|t|^{2 \alpha_{\infty}}}{2} .
$$

Note that $g_{1}(t)=g_{1}(-t), t \geq 0$, it is sufficient to prove the argument for $t \geq 0$. We distinguish three scenarios: $0<\alpha_{\infty}<1 / 2, \alpha_{\infty}=1 / 2$ and $1 / 2<\alpha_{\infty}<1$.

We first focus on $\alpha_{\infty}=1 / 2$. If $\alpha_{\infty}=1 / 2$, then

$$
g_{1}(t)=\left\{\begin{array}{cc}
1-t & 0 \leq t \leq 1 \\
0 & t \geq 1
\end{array}\right.
$$

which implies that (3.10) holds for $g_{1}(t)$.

Next we consider $0<\alpha_{\infty}<1 / 2$. For $0<t \leq 1$, the first derivative of $g_{1}$

$$
\dot{g}_{1}(t)=\alpha_{\infty}\left((1+t)^{2 \alpha_{\infty}-1}-(1-t)^{2 \alpha_{\infty}-1}-2 t^{2 \alpha_{\infty}-1}\right)<0 .
$$

Moreover, for $t>1$, by the convexity of $t^{2 \alpha_{\infty}-1}$

$$
\dot{g}_{1}(t)=\alpha_{\infty}\left((1+t)^{2 \alpha_{\infty}-1}+(t-1)^{2 \alpha_{\infty}-1}-2 t^{2 \alpha_{\infty}-1}\right)>0 .
$$


Additionally, direct calculation shows that $\lim _{t \rightarrow \infty} g_{1}(t)=0$. This means that for $0<\alpha_{\infty}<1 / 2, g_{1}(t)$ is strictly decreasing over $(0,1)$ and increasing over $(1, \infty)$ with $g_{1}(0)=1, g_{1}(1)<0$ and $\lim _{t \rightarrow \infty} g_{1}(t)=0$. This implies that for any $0<\delta<1$,

$$
\sup _{t>\delta} g_{1}(t)<1 .
$$

Thus (3.10) holds for $g_{1}$ with $0<\alpha_{\infty}<1 / 2$.

Finally, we focus on $1 / 2<\alpha_{\infty}<1$. For $0<t<1$, using the fact that $s^{2 \alpha_{\infty}-2}$ is strictly decreasing over $(0, \infty)$, we have

$$
\begin{aligned}
\dot{g}_{1}(t) & =\alpha_{\infty}\left((1+t)^{2 \alpha_{\infty}-1}-(1-t)^{2 \alpha_{\infty}-1}-2 t^{2 \alpha_{\infty}-1}\right) \\
& \leq \alpha_{\infty}\left((1+t)^{2 \alpha_{\infty}-1}-(1-t)^{2 \alpha_{\infty}-1}-(2 t)^{2 \alpha_{\infty}-1}\right) \\
& =\alpha_{\infty}\left(2 \alpha_{\infty}-1\right)\left(\int_{1-t}^{1+t} s^{2 \alpha_{\infty}-2} d s-\int_{0}^{2 t} s^{2 \alpha_{\infty}-2} d s\right)<0 .
\end{aligned}
$$

For $t>1$, by the convexity of $t^{2 \alpha_{\infty}-1}$,

$$
\dot{g}_{1}(t)=\alpha_{\infty}\left((1+t)^{2 \alpha_{\infty}-1}+(t-1)^{2 \alpha_{\infty}-1}-2 t^{2 \alpha_{\infty}-1}\right)<0 .
$$

Additionally, direct calculation shows that $\lim _{t \rightarrow \infty} g_{1}(t)=0$. Thus we have that $g_{1}(t)$ is strictly decreasing over $(0, \infty)$ with $g_{1}(0)=1$ and $\lim _{t \rightarrow \infty} g_{1}(t)=0$. Clearly, for any $0<\delta<1$,

$$
\sup _{t>\delta} g_{1}(t)<1,
$$

implying that (3.10) holds for $1 / 2<\alpha_{\infty}<1$. This completes the proofs.

Acknowledgement: P. Liu was supported by the Swiss National Science Foundation Grant 200021-175752/1. Research of K. Kosiński was conducted under scientific Grant No. 2014/12/S/ST1/00491 funded by National Science Centre.

\section{References}

N. H. Bingham, C. M. Goldie and J. L. Teugels. Regular variation, volume 27 of Encyclopedia of Mathematics and its Applications. Cambridge University Press, Cambridge (1987). ISBN 0-521-30787-2. MR898871.

A. B. Dieker. Extremes of Gaussian processes over an infinite horizon. Stochastic Process. Appl. 115 (2), 207-248 (2005). MR2111193.

K. Dębicki. Ruin probability for Gaussian integrated processes. Stochastic Process. Appl. 98 (1), 151-174 (2002). MR1884928.

K. Dębicki, S. Engelke and E. Hashorva. Generalized Pickands constants and stationary max-stable processes. Extremes 20 (3), 493-517 (2017). MR3679979.

K. Dębicki and E. Hashorva. On extremal index of max-stable stationary processes. Probab. Math. Statist. 37 (2), 299-317 (2017). MR3745388.

K. Dębicki and K. M. Kosiński. On the infimum attained by the reflected fractional Brownian motion. Extremes 17 (3), 431-446 (2014). MR3252820.

K. Dębicki and K. M. Kosiński. An Erdös-Révész type law of the iterated logarithm for reflected fractional Brownian motion. Extremes 20 (4), 729-749 (2017). MR3737383.

K. Dębicki and K. M. Kosiński. An Erdös-Révész type law of the iterated logarithm for order statistics of a stationary Gaussian process. J. Theoret. Probab. 31 (1), 579-597 (2018). MR3769825. 
K. Dębicki and P. Liu. Extremes of stationary Gaussian storage models. Extremes 19 (2), 273-302 (2016). MR3493178.

P. Erdös and P. Révész. A new law of iterated logarithm. Acta Math. Hungar. 55 (1-2), 125-131 (1990). MR1077066.

E. Hashorva, L. Ji and V. I. Piterbarg. On the supremum of $\gamma$-reflected processes with fractional Brownian motion as input. Stochastic Process. Appl. 123 (11), 4111-4127 (2013). MR3091101.

J. Hüsler and V. Piterbarg. Extremes of a certain class of Gaussian processes. Stochastic Process. Appl. 83 (2), 257-271 (1999). MR1708208.

J. Hüsler and V. Piterbarg. Limit theorem for maximum of the storage process with fractional Brownian motion as input. Stochastic Process. Appl. 114 (2), 231-250 (2004). MR2101242.

M. R. Leadbetter, G. Lindgren and H. Rootzén. Extremes and related properties of random sequences and processes. Springer Series in Statistics. Springer-Verlag, New York-Berlin (1983). ISBN 0-387-90731-9. MR691492.

P. Liu, E. Hashorva and L. Ji. On the $\gamma$-reflected processes with fBm input. Lith. Math. J. 55 (3), 402-412 (2015). MR3379034.

I. Norros. A storage model with self-similar input. Queueing Systems Theory Appl. 16 (3-4), 387-396 (1994). MR1278465.

V. I. Piterbarg. Asymptotic methods in the theory of Gaussian processes and fields, volume 148 of Translations of Mathematical Monographs. American Mathematical Society, Providence, RI (1996). ISBN 0-8218-0423-5. MR1361884.

V. I. Piterbarg. Large deviations of a storage process with fractional Brownian motion as input. Extremes 4 (2), 147-164 (2002) (2001). MR1893870.

C. Qualls and H. Watanabe. An asymptotic $0-1$ behavior of Gaussian processes. Ann. Math. Statist. 42, 2029-2035 (1971). MR0307317.

E. Reich. On the integrodifferential equation of Takács. I. Ann. Math. Statist 29, 563-570 (1958). MR0093834.

Q. M. Shao. An Erdös-Révész type law of the iterated logarithm for stationary Gaussian processes. Probab. Theory Related Fields 94 (1), 119-133 (1992). MR1189089.

F. Spitzer. Principles of random walk. The University Series in Higher Mathematics. D. Van Nostrand Co., Inc., Princeton, N.J.-Toronto-London (1964). MR0171290.

H. Watanabe. An asymptotic property of Gaussian processes. I. Trans. Amer. Math. Soc. 148, 233-248 (1970). MR0256478. 